\title{
Linear and nonlinear decay of cat's eyes in two-dimensional vortices, and the link to Landau poles
}

\author{
By M. R. TURNER AND ANDREW D. GILBERT \\ Mathematics Research Institute, School of Engineering, Computer Science and Mathematics, \\ University of Exeter, Exeter EX4 4QF, U.K.
}

(Received 20 August 2007)

This paper considers the evolution of smooth, two-dimensional vortices subject to a rotating external strain field, which generates regions of recirculating, cat's eye stream line topology within a vortex. When the external strain field is smoothly switched off, the cat's eyes may persist, or they may disappear as the vortex relaxes back to axisymmetry. A numerical study obtains criteria for the persistence of cat's eyes as a function of the strength and time-scale of the imposed strain field, for a Gaussian vortex profile.

In the limit of a weak external strain field and high Reynolds number, the disturbance decays exponentially, with a rate that is linked to a Landau pole of the linear inviscid problem. For stronger strain fields, but not strong enough to give persistent cat's eyes, the exponential decay of the disturbance varies: as time increases the decay slows down, because of the nonlinear feedback on the mean profile of the vortex. This is confirmed by determining the decay rate given by the Landau pole for these modified profiles. For strain fields strong enough to generate persistent cat's eyes, their location and rotation rate are determined for a range of angular velocities of the external strain field, and are again linked to Landau poles of the mean profiles, modified through nonlinear effects.

\section{Introduction}

Coherent vortices are an important feature of fluid flows at high Reynolds number, particularly in two dimensions, where many flows are dominated by the dynamics and interactions of these long-lived structures (Fornberg 1977; McWilliams 1984; Benzi et al. 1986; Brachet et al. 1988). Understanding the stability and dynamics of such vortices is particularly important for atmospheric applications (for example, Guinn \& Schubert 1993, Smith \& Montgomery 1995, Montgomery \& Kallenbach 1997). Many studies have considered the behaviour of an axisymmetric vortex subjected to a non-axisymmetric disturbance, which could be weak or strong. Early on, an important process of axisymmetrization was identified: a vortex may evolve dynamically to an axisymmetric state as the non-axisymmetric components of the vorticity distribution become every more finelyscaled through spiral wind-up in the underlying flow field (Melander et al. 1987). Since this spiral wind-up process does not lead to these components tending to zero point-wise (except on long, viscous time-scales), a more convenient definition of axisymmetrization is to say that the non-axisymmetric components of the stream function $\psi$, that is angular Fourier modes $\psi_{k} e^{i k \theta}$ with $k \neq 0$, tend to zero at large times. In particular the far-field flow becomes axisymmetric as time $t \rightarrow \infty$.

It was soon realised that this axisymmetrization process only occurs for sufficiently weak disturbances, and that above a certain amplitude threshold, long-lived nonlinear 
rotating states can be formed (Dritschel 1989, 1998; Koumoutsakos 1997; Rossi et al. 1997). These can take the form of multipoles; for example the addition of a pure azimuthal wave number $m=2$ vorticity distribution can cause the vortex to evolve into a tripole consisting of a vortex core and two opposite-signed satellite vortices (Rossi et al. 1997; Barba \& Leonard 2007). Another possibility is to take a stable axisymmetric vortex and expose it to an external irrotational strain field, another $m=2$ disturbance. If the strain is weak, then linear theory applies and all perturbations axisymmetrize (Briggs et al. 1970; Bassom \& Gilbert 1998; Le Dizès 2000). However for higher values, the vorticity can evolve into a pair of cat's eyes rotating about the coherent vortex core (Schecter et al. 2000; Balmforth et al. 2001; Macaskill et al. 2002). Within the cat's eyes the vorticity, which has the same sign as the core, becomes wound up and homogenized.

In the present paper we consider the evolution of a stable axisymmetric vortex, for example a Gaussian vortex, exposed to an external irrotational strain field $\psi_{\text {ext }}$, which could model the effect of other vortices in a flow, or the motion of distant boundaries. The important feature is that no vorticity is introduced into the flow by $\psi_{\text {ext }}$, and so any evolution consists of rearranging the vorticity in the original profile into a new state, axisymmetric or otherwise (insofar as viscosity may be neglected). Our aim is to determine the threshold for the formation of cat's eyes as a function of the strength and duration of the external strain field, and to compare with the studies of Le Dizès (2000) and Balmforth et al. (2001), which are based on asymptotic models. When the external strain is at a level below the threshold for the formation of cat's eyes, we determine the decay rate and how it links to the profile of the vortex: we find that as the threshold is approached, the speed of decay is reduced, and this is linked to the change in the mean, axisymmetric profile caused by nonlinear effects, as suggested by Schecter et al. (2000) and Balmforth et al. (2001).

We are also concerned with possible values of the radius $r$ at which cat's eyes may form within a coherent vortex. To this end we impose a rotating external strain field one of whose parameters is its angular velocity $\alpha_{\text {ext }}$. If we imagine a purely kinematic situation in which this strain is simply added to an axisymmetric flow field with angular velocity $\alpha_{0}(r)$, then there is a key radius $r_{\text {ext }}$ for which $\alpha_{0}\left(r_{\text {ext }}\right)=\alpha_{\text {ext }}$. At this radius the fluid particles co-rotate with the external strain, and resonant recirculating regions, cat's eyes, form. These rotate at an angular velocity $\alpha_{\text {ext }}$, with the external strain. In a properly dynamical simulation with an initial angular velocity $\alpha_{0}(r)$ the picture is more complicated, as the advected vorticity then affects the flow field, but the issue arises: if a transient external strain field is imposed with angular velocity $\alpha_{\text {ext }}$, can cat's eyes be generated at a radius $r_{\text {ext }}$ that then persist at late times when the external forcing is negligible? If not, where do they form?

To understand some of the dynamical aspects, we need to introduce the concept of a 'quasi-mode'. In the limit of a weak $m=2$ disturbance to a Gaussian vortex, as the vortex axisymmetrizes, the far-field streamfunction shows an initial phase of exponential damping with decay rate $\gamma_{\mathrm{qm}}<0$ and angular velocity $\alpha_{\mathrm{qm}}$. This behaviour is termed a quasi-mode (Briggs et al. 1970; Schecter et al. 2000; Balmforth et al. 2001) and has been observed numerically (Bassom \& Gilbert 1998) and in experiments (Schecter et al. 2000). The dynamics of these quasi-modes is discussed in more detail in $\S 3.1$, but for now we may consider the dynamics of a Gaussian vortex as possessing a natural damped mode, with angular velocity $\alpha_{\mathrm{qm}}$. In our simulations we vary $\alpha_{\text {ext }}$ to understand how the angular velocity of the external forcing interacts with the angular velocity of this natural quasi-mode, and whether generally we form persistent cat's eyes at the radius $r_{\text {ext }}$ linked to the forcing, or at $r_{\mathrm{qm}}$ linked to the quasi-mode, or somewhere else. The outcome is 
not obvious, as above the threshold needed to generate cat's eyes, nonlinearity will also change the mean profiles of the vorticity and angular velocity.

The structure of this paper is as follows. In $\S 2$, we formulate the problem, and present diagnostics for our simulations. Section 3 gives numerical results for the Gaussian vortex when the external strain has the same angular velocity as the quasi-mode. We observe that persistent cat's eyes form for sufficiently strong external strain fields, and map out the threshold for their formation. Section 4 is concerned with computing the angular velocity $\alpha_{\mathrm{qm}}$ and decay rate $\gamma_{\mathrm{qm}}$ for a quasi-mode on a general vortex profile, by isolating a Landau pole numerically (Schecter et al. 2000). In section 5 we vary the angular velocity of the external strain field and determine where cat's eyes form, and their rotation frequency. Again our results may be understood by determining Landau poles for the resulting mean profiles. Our concluding remarks are found in $\S 6$.

\section{Formulation}

A two-dimensional vortex with vorticity $\omega$ and stream function $\psi$ in an irrotational, externally imposed flow with stream function $\psi_{\text {ext }}$ is governed by the dimensionless vorticity equation

$$
\partial_{t} \omega-J\left(\psi+\psi_{\text {ext }}, \omega\right)=R^{-1} \nabla^{2} \omega, \quad \nabla^{2} \psi=-\omega, \quad \nabla^{2} \psi_{\text {ext }}=0,
$$

where in polar coordinates $J(a, b) \equiv r^{-1}\left(\partial_{r} a \partial_{\theta} b-\partial_{\theta} a \partial_{r} b\right)$ and the velocity field is given by $\left(\frac{1}{r} \psi_{\theta},-\psi_{r}\right)$. The stream function $\psi$ is permitted to grow no faster than $\ln r$ for large $r$, whereas $\psi_{\text {ext }}$, as a harmonic function, increases in powers of $r$. We are interested in the inviscid limit of large Reynolds number $R$. There are many processes that can occur on time and space scales linked to powers of $R$, for example the destruction of fine scales of vorticity within the vortex core, or within individual cat's eyes, on the sheardiffuse time scale of $O\left(R^{1 / 3}\right)$ (see Moffatt \& Kamkar 1983, Rhines \& Young 1983, Bernoff \& Lingevitch 1994, Bajer et al. 2001). However, unless we mention otherwise, with the diagnostics we use the results presented are essentially inviscid, taken at sufficiently large $R$ that they do not show appreciable sensitivity to changes in the value of $R$, for example from $10^{7}$ to $10^{6}$.

We start with an axisymmetric vortex $\omega=\Omega_{0}(r), \psi=\Psi_{0}(r)$ at $t=0$, usually the Gaussian vortex defined by

$$
\Omega_{0}(r)=(4 \pi)^{-1} e^{-r^{2} / 4} .
$$

This vortex has angular velocity given by

$$
\alpha_{0}(r)=-\frac{1}{r} \frac{\partial \Psi_{0}}{\partial r}=\frac{1}{r^{2}} \int_{0}^{r} s \Omega_{0}(s) d s=\frac{1}{2 \pi r^{2}}\left(1-e^{-r^{2} / 4}\right) .
$$

The structure of the Gaussian vortex in (2.2) is not always typical: vortices observed, in two-dimensional turbulence for example, often have sharp edges arising from processes such as vortex stripping (Legras \& Dritschel 1993). We therefore also consider members of a family of tanh vortices used by Hall et al. (2003), with vorticity distribution

$$
\Omega_{0}(r)=\frac{1}{4 \pi} \frac{1-\tanh \left[\left(r^{2}-\sigma^{2}\right) / 4(1-\sigma)\right]}{1+\tanh \left[\sigma^{2} / 4(1-\sigma)\right]},
$$

and angular velocity

$$
\alpha_{0}(r)=\frac{1}{4 \pi r^{2}\left(1+\tanh \left[\sigma^{2} / 4(1-\sigma)\right]\right)}\left[\frac{r^{2}}{2}-2(1-\sigma) \ln \left(\frac{\cosh \left[\left(r^{2}-\sigma^{2}\right) / 4(1-\sigma)\right]}{\cosh \left[\sigma^{2} / 4(1-\sigma)\right]}\right)\right] .
$$




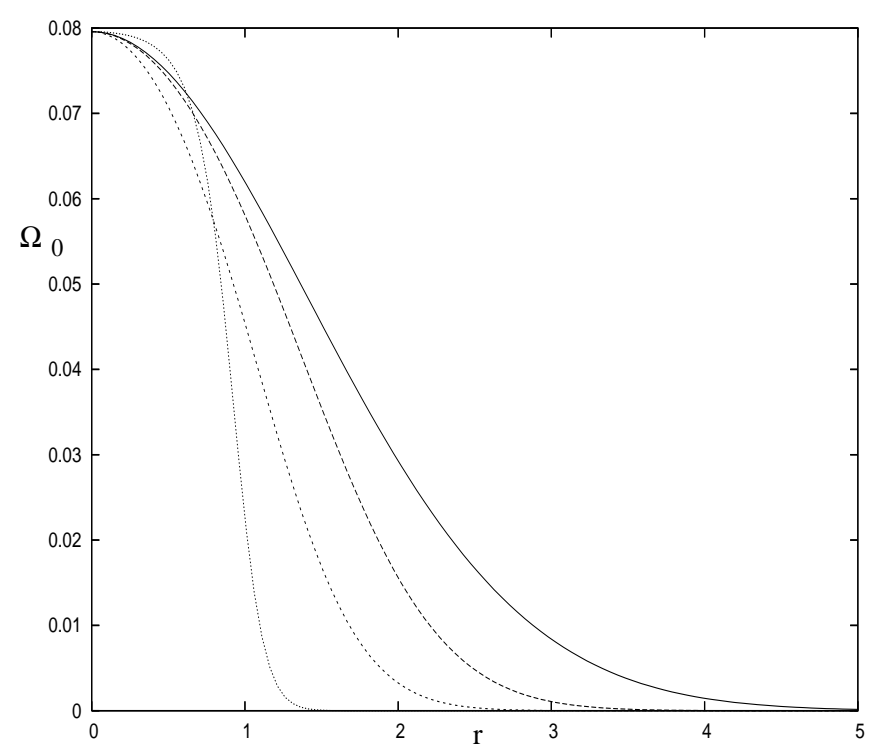

Figure 1. From right to left, the Gaussian vortex (2.2) and the tanh vortex (2.4) for $\sigma=0.1,0.5$ and 0.9 .

The members of this family vary from a broad vortex to a top hat or Rankine vortex as $\sigma$ varies from 0 to 1 . A plot of (2.4) for $\sigma=0.1,0.5$ and 0.9 , along with the Gaussian vortex $(2.2)$ can be seen in figure 1.

Starting with one of the initial conditions $(2.2)$ or $(2.4)$, an external flow $\psi_{\text {ext }}$ with azimuthal wavenumber $m \neq 0$ is switched on for $t>0$ and forces the vortex into a nonaxisymmetric state. We will always take $m=2$, in what follows; however we will often leave the quantity $m$ in our equations to show their mathematical structure. The case $m=1$ is a pure translation mode, giving no dynamical effects and so of no interest here, while we expect similar results would be obtained for any value of $m \geq 2$. Furthermore in a multipole expansion of a general external irrotational flow, $m=2$ would be the dominant term giving dynamical effects internal to the vortex.

The subsequent evolution of $\omega$ and $\psi$ is then followed by time-stepping (2.1) with the solution written as a (truncated) sum of Fourier harmonics

$$
\omega(r, \theta, t)=\sum_{k=-\infty}^{\infty} \omega_{k}(r, t) e^{i k \theta}, \quad \psi(r, \theta, t)=\sum_{k=-\infty}^{\infty} \psi_{k}(r, t) e^{i k \theta} .
$$

For $m=2$ the external, irrotational stream function $\psi_{\text {ext }}$ is pure strain, taking the form,

$$
\psi_{\text {ext }}(r, \theta, t)=\phi(t) r^{m} e^{i m \theta}+\text { c.c. }
$$

The complex function $\phi(t)$ determines the time-dependent strength and orientation of the strain field and we consider a form given by three parameters $\left(A, T, \alpha_{\text {ext }}\right)$,

$$
\phi(t)=(A t / T) e^{-t^{2} / 2 T^{2}} e^{-i m \alpha_{\mathrm{ext}} t} .
$$

The variable $A$ denotes the amplitude of the forcing, $T$ denotes some large turn off time, and $\alpha_{\text {ext }}$ is the angular velocity of the forcing. This forcing is similar to that used by Balmforth et al. (2001), although our definitions of $A$ and $T$ are not directly comparable with theirs. Note that the external strain fields we impose are not strong enough for the process of vortex stripping to occur (Legras \& Dritschel 1993); in the vicinity of 
the vortex, our flows are always dominated by the rotational component of the flow, generated by the vortex.

In order to measure the strength of the disturbance to the vortex, we define the $m^{\text {th }}$ multipole moment of the vorticity by

$$
Q_{m}(t)=\int_{0}^{\infty} r^{m+1} \omega_{m}(r, t) d r \quad(m \geq 1) .
$$

The measure $Q_{m}(t)$ is related to the far-field form of the stream function, with

$$
2 m \psi_{m}(r, t) Q_{m}(t) r^{-m} \quad(r \rightarrow \infty)
$$

(see, e.g., Bassom \& Gilbert 1998).

Another useful measure of an $m=2$ disturbance to a vortex is the instantaneous aspect ratio (major to minor axes) of the approximately elliptical streamlines at the origin (Balmforth et al. 2001). Working in a frame that co-rotates with the external strain, the stream function at the origin takes the approximate form

$$
\psi_{\text {co }}(r, \theta, t) \simeq \text { const. }+r^{2}\left(a+\alpha_{\text {ext }}\right)+b r^{2} e^{2 i \theta}+\text { c.c. }+O\left(r^{3}\right),
$$

which may be written in rotated Cartesian coordinates $\left(x^{\prime}, y^{\prime}\right)$ as

$$
\psi_{\mathrm{co}}(r, \theta, t)=\text { const. }+\left(a+\alpha_{\text {ext }}+2|b|\right) x^{\prime 2}+\left(a+\alpha_{\text {ext }}-2|b|\right) y^{\prime 2}+\cdots .
$$

We now use the fact that

$$
a=\lim _{r \rightarrow 0} \frac{1}{2} r^{-1} \partial_{r} \psi_{0}(r, t), \quad b=\lim _{r \rightarrow 0} r^{-2} \psi_{2}(r, t),
$$

to obtain the aspect ratio for near-circular stream lines ( $b$ small) in the form

$$
\nu(t)=\lim _{r \rightarrow 0} \frac{r\left|\partial_{r} \psi_{0}(r, t)+2 \alpha_{\mathrm{ext}} r\right|+2\left|\psi_{2}(r, t)\right|}{r\left|\partial_{r} \psi_{0}(r, t)+2 \alpha_{\mathrm{ext}} r\right|-2\left|\psi_{2}(r, t)\right|} .
$$

Note that as $r \rightarrow 0$, there is no singularity in (2.12), but when calculating $\nu$ numerically, we encounter inaccuracy close to the origin. Therefore we extrapolate to the origin through the relatively flat part of the curve, which minimises this problem.

We choose to measure this aspect ratio in a frame of reference rotating with the external strain, although other choices could be made. For example a frame co-rotating with the phase of $Q_{2}(t)$ or a non-rotating frame produce qualitatively similar results. We could avoid the necessity of choosing a rotating frame by using the contours of constant vorticity, rather than stream function, to calculate $\nu(t)$, however we found that the fine scale structure in the vorticity made this procedure impractical. Both $Q_{2}(t)$ and $\nu(t)$ give useful measures of the non-axisymmetric $m=2$ structure on the vortex, and indicate whether the vortex is relaxing to an axisymmetric configuration (signalled by $Q_{2} \rightarrow 0$ and $\nu \rightarrow 1$ ), or retaining persistent cat's eyes.

\section{Numerical results: threshold for cat's eye formation}

In this section we present results of fully nonlinear simulations of (2.1) for the evolution of the Gaussian vortex (2.2). The external forcing is determined by the parameters $\left(A, T, \alpha_{\text {ext }}\right)$. The imposed angular velocity of the strain field $\alpha_{\text {ext }}$ is a key parameter in our study. We also recall that given an initial angular velocity profile $\alpha_{0}(r)$ it is at the radius $r_{\text {ext }}$ given by $\alpha_{0}\left(r_{\text {ext }}\right)=\alpha_{\text {ext }}$, where particles rotate in concert with the external strain field, that the external field has a strong effect, at least initially before nonlinear effects take hold. 


\subsection{Quasi-mode properties}

The other key angular velocity in the system is that of the quasi-mode, $\alpha_{\mathrm{qm}}$; we now pause to outline some of the properties of this mode. As discussed in the introduction, following a weak, instantaneous external strain of a Gaussian vortex, there is an exponential decay of fluctuations at a rate $\gamma_{\mathrm{qm}}$, as measured by the moment $Q_{m}(t)$ with $m=2$. Also $Q_{m}(t)$ has a frequency $-m \alpha_{\mathrm{qm}}$ corresponding to an angular velocity $\alpha_{\mathrm{qm}}$. This exponential behaviour is suggestive of a normal mode, but it can be shown that these cannot exist on a Gaussian vortex, and what is seen is really a cooperative effect of the continuous spectrum (Briggs et al. 1970). The resulting quasi-mode can be thought of informally as a normal mode that is modified by the fine-scaling of vorticity at a critical radius, $r_{\mathrm{qm}}$ (Balmforth et al. 2001). Together with this exponential decay of $Q_{m}$ is also a transfer of enstrophy from fluctuations to the mean profile (Bassom \& Gilbert 1999).

We combine the decay rate and the angular velocity to give the dependence $Q_{m}(t) \propto$ $e^{-i p_{\mathrm{qm}} t}$ where the complex growth rate $-i p_{\mathrm{qm}}=\gamma_{\mathrm{qm}}-i m \alpha_{\mathrm{qm}}$ can be calculated by analytic continuation (see $\S 4$ ) and emerges from the continuous spectrum as a Landau pole. Schecter et al. (2000) compute this numerically for a Gaussian vortex with $m=2$ to obtain, rescaled in line with our conventions below,

$$
\begin{gathered}
-i p_{\mathrm{qm}} \equiv \gamma_{\mathrm{qm}}-i m \alpha_{\mathrm{qm}} \simeq(4 \pi)^{-1}(-0.079-0.222 i), \\
\gamma_{\mathrm{qm}} \simeq-0.0063, \quad \alpha_{\mathrm{qm}} \simeq 0.0089 .
\end{gathered}
$$

(Note that Schecter et al. (2000) in fact obtain a slightly different value $\alpha_{\mathrm{qm}}=0.0090$ as they work in a bounded domain with constant $\psi$ imposed at the radius $r_{0}=10$ in our units, rather than the infinite domain we use in $\S 4$.)

The quasi-mode angular velocity $\alpha_{\mathrm{qm}}$ also singles out the critical radius $r_{\mathrm{qm}}$, where fluid particles co-rotate, given by

$$
\alpha_{0}\left(r_{\mathrm{qm}}\right)=\alpha_{\mathrm{qm}}, \quad r_{\mathrm{qm}} \simeq 4.22 .
$$

The complex growth rate $-i p_{\mathrm{qm}}$ of the quasi-mode, and so of weak disturbances to a vortex, depends only on the vorticity profile $\Omega_{0}(r)$, not on the nature of the disturbance. The decay rate $\gamma_{\mathrm{qm}}$ is sensitive to any changes in this vorticity distribution near to $r_{\mathrm{qm}}$, as we shall see.

The numerical code uses only even Fourier modes in the $\theta$ direction; typical runs are truncated at $|k|=256$ in (2.6) and use 1500 radial grid points, with the maximum value of $r$ as $r_{0}=15$. The code uses second order finite differences for radial derivatives. The diffusion term is integrated using the Crank-Nicolson method and nonlinear terms using the Adam-Bashforth method.

At the origin we fix $\psi_{k}=0$ for all $k$, but at $r=r_{0}$ we take

$$
\psi_{0}=-(2 \pi)^{-1} \ln r_{0}, \quad \partial_{r} \psi_{k}+r_{0}^{-1}|k| \psi_{k}=0 \quad(k \neq 0) .
$$

The second of these conditions ensures that $\psi_{k}$ matches to the irrotational flow $\psi_{k} \propto r^{-|k|}$ for $r \geq r_{0}$. As all the wavenumbers $k$ are multiples of $m=2$, the flow velocities scale as $r$ near the origin, and so there is no need for special numerical treatment of the pole $r=0$.

\subsection{Gaussian vortex: $\alpha_{\mathrm{ext}}=\alpha_{\mathrm{qm}}$}

In this section we consider the evolution of a Gaussian vortex when the angular velocity of the forcing $\alpha_{\text {ext }}=\alpha_{\mathrm{qm}}$ and so $r_{\text {ext }}=r_{\mathrm{qm}}$. We should stress again that the angular velocity $\alpha_{\text {ext }}$ of the forcing is a free parameter, and we are now choosing it to be the 


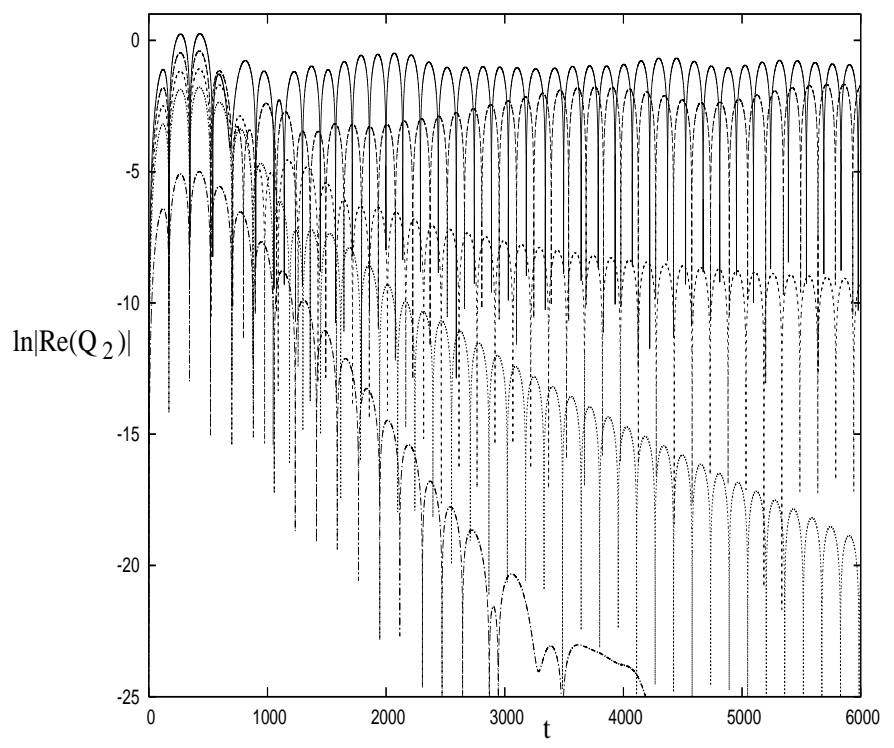

Figure 2. From bottom left to top right, $\ln \left|\operatorname{Re}\left(Q_{2}(t)\right)\right|$ plotted against time $t$, with $\alpha_{\text {ext }}=\alpha_{\text {qm }}$, $T=200, R=10^{7}$ for $A=0.000413$ (dot-dash), $A=0.0103$ (dot), $A=0.0206$ (dash), $A=0.0413$ (wide dash), and $A=0.0825$ (solid).

same as the quasi-mode angular velocity $\alpha_{\mathrm{qm}}$. We then expect principally to excite this structure on the vortex, as in the asymptotic framework of Balmforth et al. (2001).

For the Gaussian vortex given in (2.2), figure 2 shows a plot of $\ln \left|\operatorname{Re}\left(Q_{2}\right)\right|$, as a function of time, for the case

$$
R=10^{7}, \quad T=200, \quad \alpha_{\mathrm{ext}}=\alpha_{\mathrm{qm}}=0.0089 .
$$

The external strain field becomes effectively negligible around $t=1000$ for this value of $T$. The downward spikes of $\ln \left|\operatorname{Re}\left(Q_{2}\right)\right|$ in the plot correspond to points where $\operatorname{Re}\left(Q_{2}\right)$ is zero. The full quantity $Q_{2}(t)$ evolves as a complex function with quickly changing phase. However the amplitude of $Q_{2}(t)$ and the rate of change of phase vary relatively slowly, especially at large times, and we can define a time-dependent exponential decay rate $\gamma_{Q}$ and angular velocity $\alpha_{Q}$ by

$$
\gamma_{Q}(t)=\frac{d}{d t} \log \left|Q_{m}(t)\right|, \quad-m \alpha_{Q}(t)=\frac{d}{d t} \arg Q_{m}(t) .
$$

These are also the decay rate and angular velocity of the far-field flow for the $m=2$ mode.

The amplitudes $A$ of the external strain used in figure 2 are given in the caption. For the smallest amplitude, giving the lowest curve of the five, $Q_{2}(t)$ falls off exponentially, with a decay rate $\gamma_{Q} \simeq-0.0063$ and angular velocity $\alpha_{Q} \simeq 0.0089$ (measured at time $t=1500)$ that are in agreement with (3.2). For this value of $A$, at around $t=3000, Q_{2}(t)$ then moves into a second regime described by Bassom \& Gilbert (1998), where the decay of $Q_{2}$ is dominated by the large- $r$ form of the vorticity and is slower than exponential. However these later regimes (when the disturbance is already very weak) do not concern us here.

Increasing the amplitude of $A$ gives the second and third curves in figure 2; the quantity $Q_{2}(t)$ still decays rapidly at times $t>1000$ when the forcing is negligible. However now the frequency and decay rate are different from those for the smallest value of $A$ 
(a)

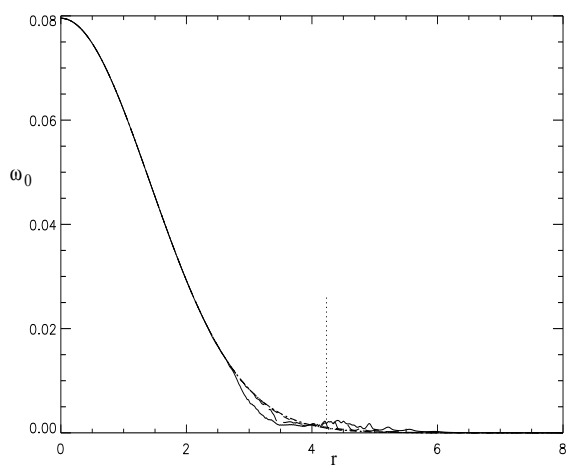

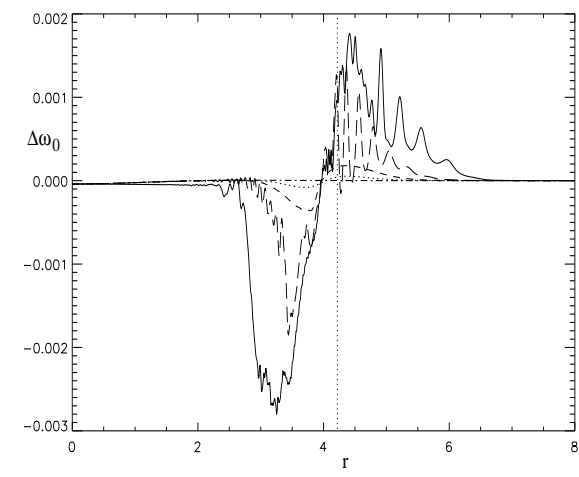

(b)

FIgURE 3. Profiles of (a) $\omega_{0}$ and (b) $\Delta \omega_{0}$ showing how the initial vorticity profile is modified, at $t=5000$, for the conditions in figure 2 with corresponding values of $A$ and line styles. The vertical dotted line indicates the value of $r_{\mathrm{qm}}$ from (3.3).

(lowest curve) given by the Landau pole in (3.2); particularly noticeable is the curvature indicating that the decay is slowing down with time, i.e., the negative quantity $\gamma_{Q}(t)$ is increasing.

The reason for this effect has been suggested by Schecter et al. (2000) and Balmforth et al. (2001): with increasing nonlinearity there is an effect on the mean vorticity profile and with increasing time the profile $\omega_{0}(r, t)$ in (2.6) differs from the initial one $\Omega_{0}(r)$ in (2.2). This effect is particularly strong in the vicinity of $r_{\mathrm{qm}}$ where the profile tends to be flattened because of the resonant forcing of the flow from the external strain in the case $\alpha_{\mathrm{qm}}=\alpha_{\text {ext }}$. The changes in profile can be seen in figure 3 which shows (a) $\omega_{0}(r, t)$ and the more informative quantity (b) $\Delta \omega_{0}(r, t) \equiv \omega_{0}(r, t)-\Omega_{0}(r)$. The local flattening at $r \simeq r_{\mathrm{qm}}$ has the effect of changing the position of the Landau pole as time proceeds, and so giving a slowly varying decay rate. We will confirm this interpretation by computing Landau poles in section 4.

For still larger values of the amplitude $A$ (upper two curves in figure 2) the quantity $Q_{2}(t)$ does not decay for large times; after an initial dip it increases to a constant level, with superposed, somewhat irregular oscillations. During the increasing phase the cat's eyes generated by the forcing grow, instead of becoming thinner and weaker as in the cases of smaller $A$, and vorticity in the vicinity of $r_{\mathrm{qm}}$ overturns to form a long-lived non-axisymmetric distribution. This is found by Balmforth et al. (2001) within their asymptotic framework and confirmed in the experiments of Schecter et al. (2000).

Figure 3 shows how the amplitude of the forcing $A$ modifies the mean vorticity profile $\omega_{0}$ for the conditions in figure 2 at $t=5000$. We see that for the two largest values of $A$ (solid, wide dash) the vorticity profile is modified significantly around $r_{\mathrm{qm}}$, and these profiles contain fine structure from vorticity fluctuations in the cat's eyes, also seen in Balmforth et al. (2001). For these two largest amplitudes the mean vorticity profile is flattened and the vorticity homogenised in the vicinity of the critical radius $r_{\mathrm{qm}}$, here marked with a vertical dotted line. For the three smaller forcing amplitudes, the profiles contain no fine structure, and in fact in figure 3(a), the Gaussian profile appears almost unchanged; however from figure 2, it appears that even such apparently insignificant changes can still have an appreciable effect on the decay rate of $Q_{2}(t)$. We note that as $A$ decreases the width of the cat's eyes decreases and so a thinner flat region is obtained around $r=r_{\mathrm{qm}}$.

The cat's eyes generated can be seen by plotting contours of the co-rotating stream 

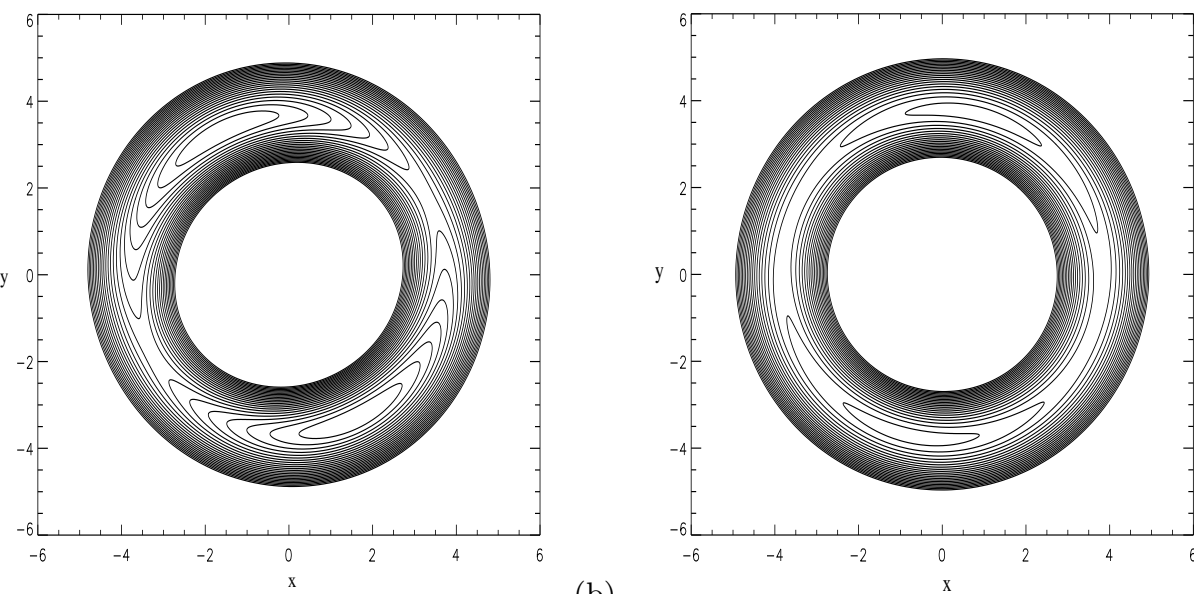

(a)

(b)

FigURE 4 . The co-rotating stream function, $\psi_{\mathrm{co}}=\psi+\alpha_{Q} r^{2}$ at $t=3000$, for $R=10^{7}, \alpha_{\mathrm{ext}}=\alpha_{\mathrm{qm}}$ and (a) $A=0.0825$, and (b) $A=0.0413$. These figures were completed using 40 equally spaced contours.
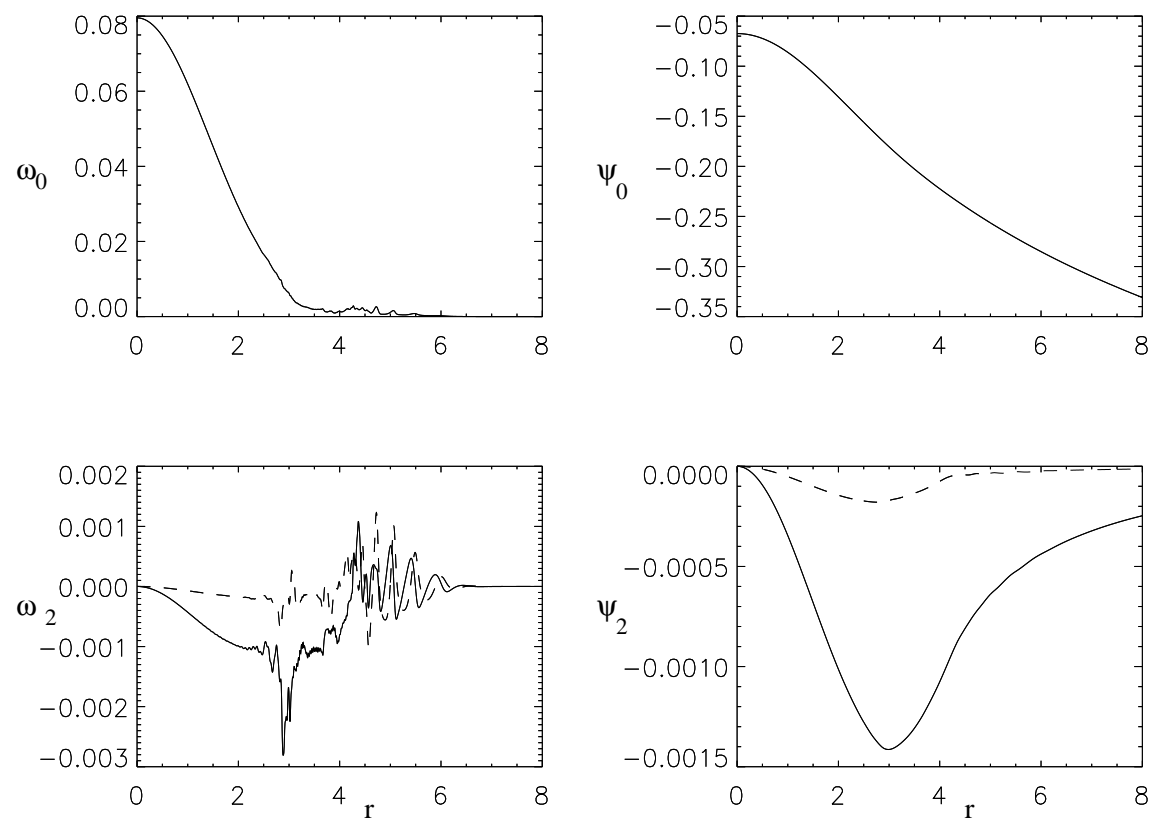

Figure 5. Profiles of $\omega_{0}(r), \psi_{0}(r)$, and the real (solid) and imaginary (dash) parts of $\omega_{2}(r)$ and $\psi_{2}(r)$ for $R=10^{7}$ and $A=0.0825$ at $t=4000$, with $\alpha_{\text {ext }}=\alpha_{\mathrm{qm}}$.

function $\psi_{\text {co }}=\psi+\alpha_{Q} r^{2}$ where $\alpha_{Q}$ depends on $t$. In this co-rotating frame, the cat's eyes can be seen as closed contours around the critical radius at $r=r_{\mathrm{qm}}$. The panels in figure 4 plot the co-rotating stream function at $t=3000$ for the two largest values of $A$. We note again that as the amplitude of the forcing is reduced, the width of the cat's eyes decreases.

In figures 5 and 6 , we examine the form of $\omega_{0}, \psi_{0}$ and the real and imaginary parts of 

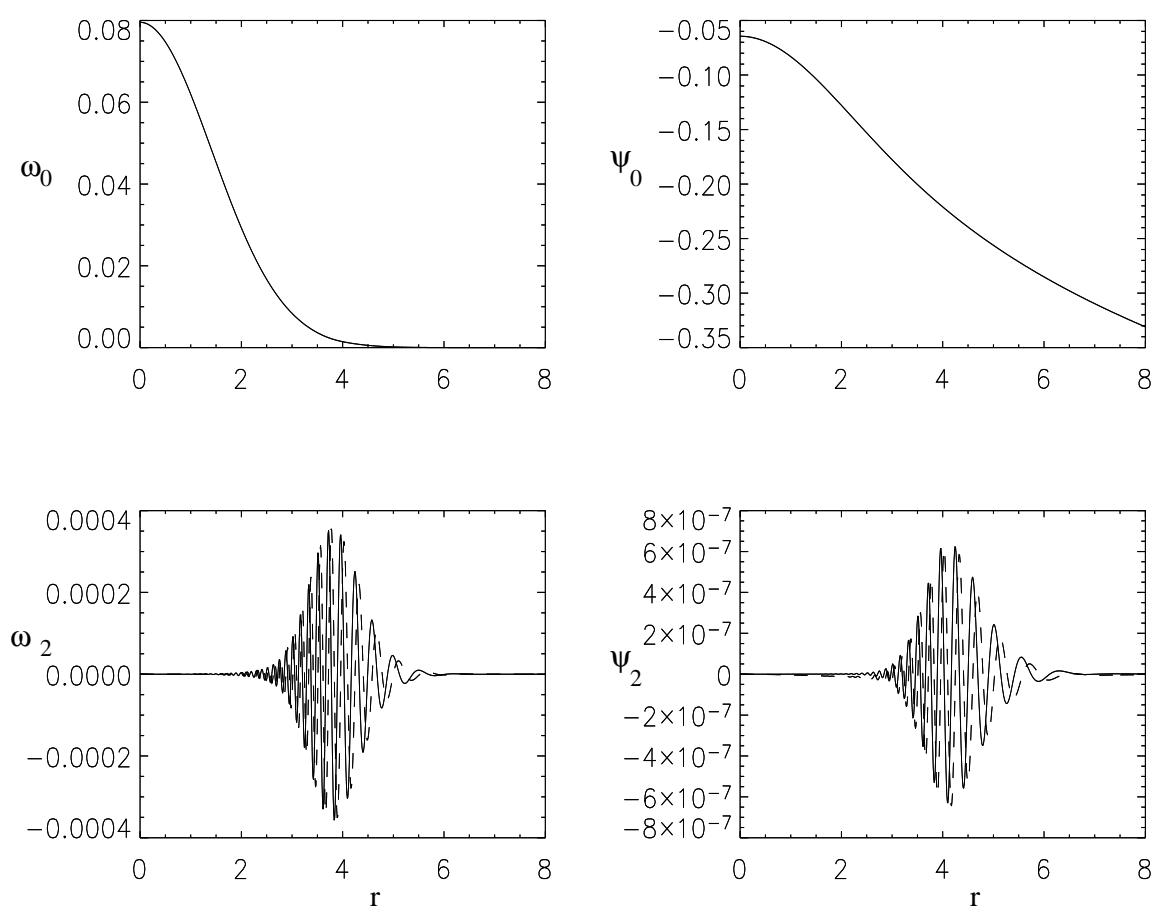

Figure 6 . As figure 5, but with $A=0.0103$.

$\omega_{2}$ and $\psi_{2}$ at $t=4000$ for a large amplitude, $A=0.0825$, and a smaller amplitude, $A=$ 0.0103 , respectively. For the large amplitude case in figure 5 , cat's eyes have maintained themselves from the initial forcing, and we see that the profile of $\omega_{0}$ has flattened out around $r=r_{\mathrm{qm}} \simeq 4.2$, there being some fine scale structure from the cat's eyes visible. The $m=2$ mode $\omega_{2}$ shows this fine structure again, but there is none in the perturbation stream function $\psi_{2}$. The fine scale structure seen in the perturbation vorticity $\omega_{2}$ is still visible even when the number of radial grid points is increased to $M=9000$ and is not a numerical artefact. Note that $\omega_{2}$ is quite smooth for $r$ less than about 2, and what we are seeing may be thought of as a normal mode on the core of the vortex, coupled to fine structure for $r$ greater than about 2.5.

In figure 6 , the forcing amplitude is small enough so that $Q_{2}$ decays and the thickness of the cat's eyes decreases with time; by $t=4000$ they are extremely thin. In this case, the mean stream function $\psi_{0}$ again appears unaffected by the perturbation, as does the mean vorticity $\omega_{0}$ which is very close to its initial Gaussian form. The structure of the perturbation vorticity $\omega_{2}$ and perturbation stream function $\psi_{2}$ are both very similar, and are dominated by the spiral wind-up of fluctuations (Bassom \& Gilbert 1998). Note from these figures, 5 and 6 , that the magnitude of the perturbation vorticity $\omega_{2}$ differs by a factor of 10 between the two cases, whereas the magnitude of the perturbation stream function $\psi_{2}$ differs by a factor of 2000 . This highlights the contrast between the relaxation to axisymmetry of the stream function in the second, weakly forced case, and the presence of long-lived non-axisymmetric cat's eyes in the first, strongly forced case.

Another useful diagnostic here is the aspect ratio $\nu(t)$, defined in (2.12), of the stream lines at the origin. Figure 7 (a) presents $\nu(t)-1$ for the same data as figure 2 . This shows 


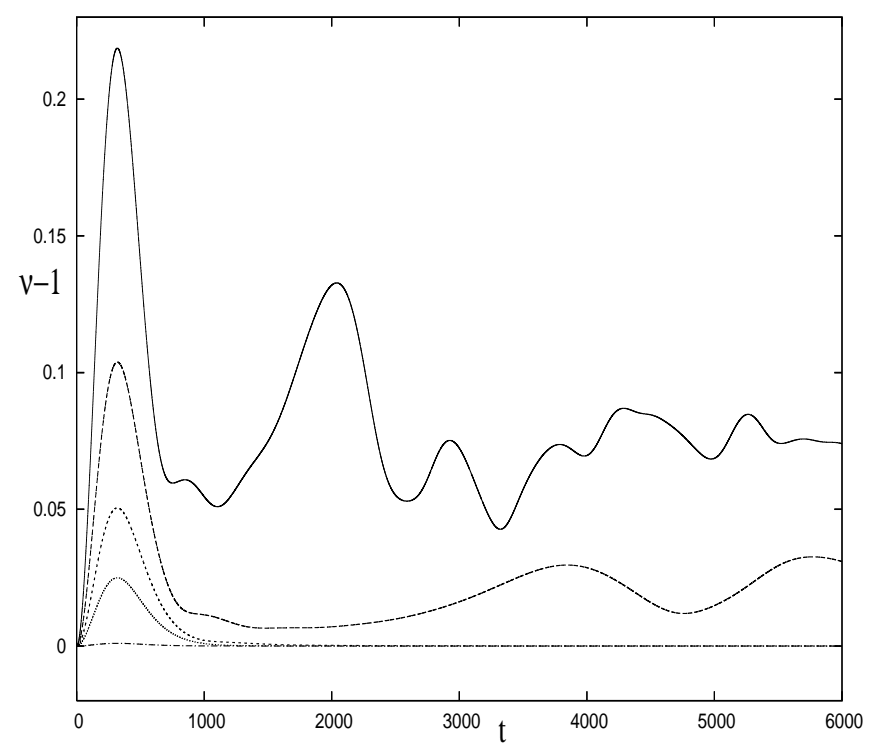

Figure 7. Aspect ratio of the streamlines at the origin: $\nu(t)-1$ is shown for $R=10^{7}$, $\alpha_{\text {ext }}=\alpha_{\mathrm{qm}}$ and, from bottom to top, increasing values of $A$ as in figure 2 .

that for the three smallest forcing amplitudes, the vortex does return to axisymmetry after the forcing has become negligible around $t=1000$. However for the two larger amplitudes we see that the streamlines remain elliptical at the origin. For the $A=0.0825$ case, a large peak in $\nu(t)-1$ occurs at around $t=2000$, when the cat's eye motion first turns over, and is equivalent to the first 'bounce' noted by Balmforth et al. (2001). Note that a $10 \%$ distortion of the streamlines at the origin in the initial phase is enough to prevent axisymmeterization, but the distortion then goes down to $1-3 \%$, though does not settle on the time-scale of the simulation. These figures are in agreement with the $5-10 \%$ estimate given by Balmforth et al. (2001).

It is clear then that there is an amplitude threshold for the formation of long-lived cat's eyes. However we lack a convenient and completely unambiguous numerical criterion for their formation, because of the ups and downs of the $Q_{2}$ curves as seen in figure 2. A similar issue is faced by Balmforth et al. (2001) who use a criterion based on the occurrence of a first bounce in the stream function within some given time. We have instead focused on the behaviour of $\left|Q_{2}\right|$, and its growth or decay after the initial rearrangement of vorticity. We use as our diagnostic:

$$
\text { if } \frac{d\left|Q_{2}\right|}{d t}>0 \text { at any point for } 3000<t<5000,
$$

then we say that long-lived cat's eyes have formed. If however it is monotonically decreasing in this region, then we take it that the vortex is relaxing to axisymmetry.

Figure 8 shows a plot in the $(A, T)$-plane indicating points where the diagnostic (3.7) is satisfied for the Gaussian vortex, compiled from a series of runs with $\alpha_{\mathrm{ext}}=\alpha_{\mathrm{qm}}$ and $R=10^{7}$. Above the contour, the cat's eyes generated in the Gaussian vortex persist and grow, and below this contour, the cat's eyes die away. In the case of a Gaussian vortex, as $T \rightarrow 0$ the forcing approaches the limit of an instantaneous kick, and the threshold value of $A \simeq 0.033$.

Balmforth et al. (2001) produce a similar figure, their figure 13(a); however their result is for an asymptotic model of a compact vortex with a weak skirt while ours is for the 


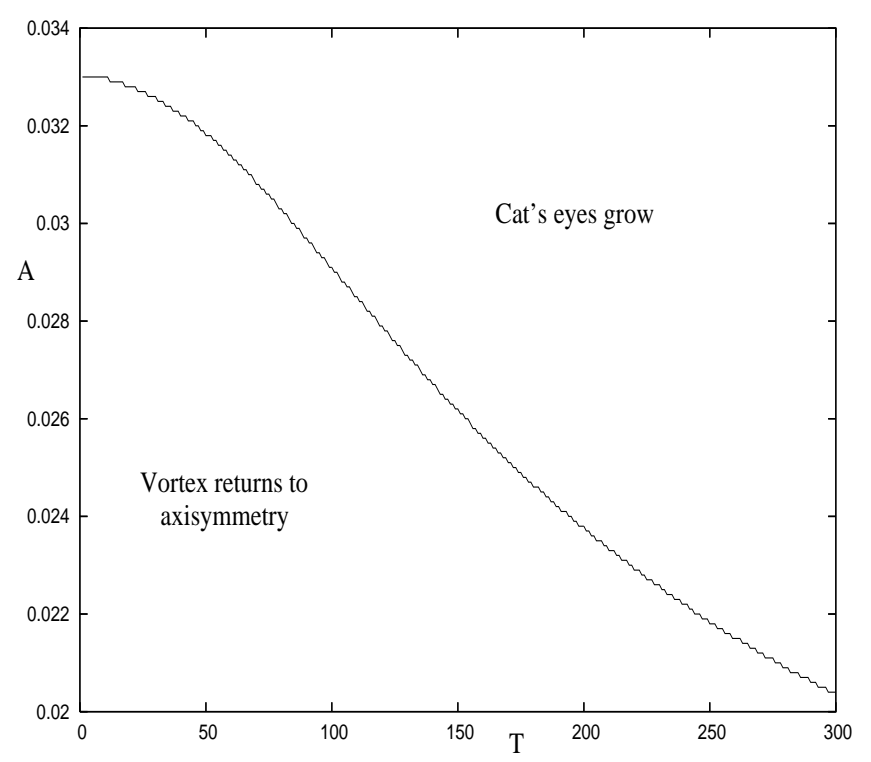

Figure 8. Plot of $A$ against $T$ showing the threshold above which cat's eyes grow, and below which the vortex returns to axisymmetry using the diagnostic (3.7) for a Gaussian vortex at $R=10^{7}$ with $\alpha_{\text {ext }}=\alpha_{\mathrm{qm}}$.

Gaussian vortex. Although their diagnostic is different, and their definitions of $A$ and $T$ also differ from ours, the two plots are similar, with a drop in the threshold value of $A$ as $T$ increases.

We have not tried to pursue the issue of the behaviour of the threshold $A$ as $T$ increases, as long-time runs at low amplitudes and high Reynolds numbers become numerically impractical. Within their model Balmforth et al. (2001) study the threshold based on a criterion of when the function $\Delta \omega_{0}\left(Z^{\infty}\right.$ in their work) first develops a turning point. For their forcing they determine this contour in the $(A, T)$-plane analytically, and so suggest the scaling of threshold amplitude $A=O(1 / T) \longrightarrow 0$ as $T \longrightarrow \infty$. Our threshold also falls off with increasing $T$, but we have not attempted to determine a power law.

In the limit of large $T$, the evolution in the steady state problem of constant amplitude forcing becomes relevant, and this is currently under investigation (see also Lingevitch \& Bernoff (1995) for the linear problem at finite Reynolds number). Here we note that at the larger amplitudes considered in the present paper, the structure of the vortex continues to evolve on time-scales rather greater than those considered here, for example as measured by $\left|Q_{2}(t)\right|$ which shows slow, irregular oscillations.

\subsection{Formation of cat's eyes for members of the tanh vortex family}

The simulations of $\S 3.2$ and $\S 5$ for an initial Gaussian vortex can also be applied to other vortices. In this section we take a brief look at what happens when the tanh vortex given by (2.4) is subjected to a forcing with $\alpha_{\text {ext }}=\alpha_{\mathrm{qm}}$.

Figure 9 shows plots of $\ln \left|\operatorname{Re}\left(Q_{2}\right)\right|$ at the five values of $A$ used in figure 2, for the tanh vortex (2.4) with $\sigma=$ (a) 0.1 , (b) 0.5 and (c) 0.9 . For these vortices the values of $\alpha_{\mathrm{qm}}$ and $\gamma_{\mathrm{qm}}$ are obtained using the Landau pole calculation of $\S 4$, and the resonant radii $r_{\mathrm{qm}}$ 
(a)

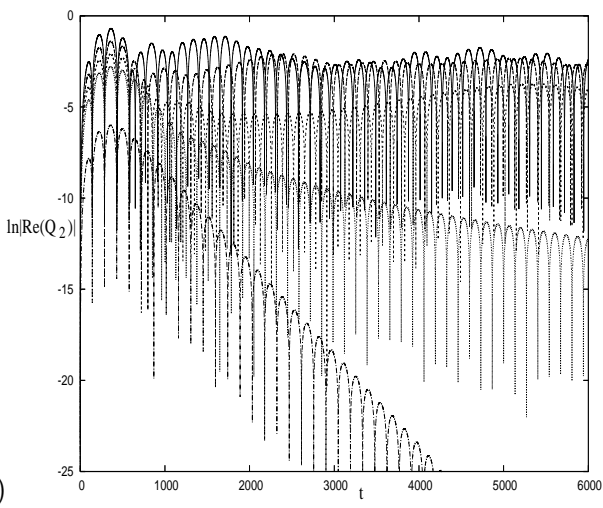

(b)

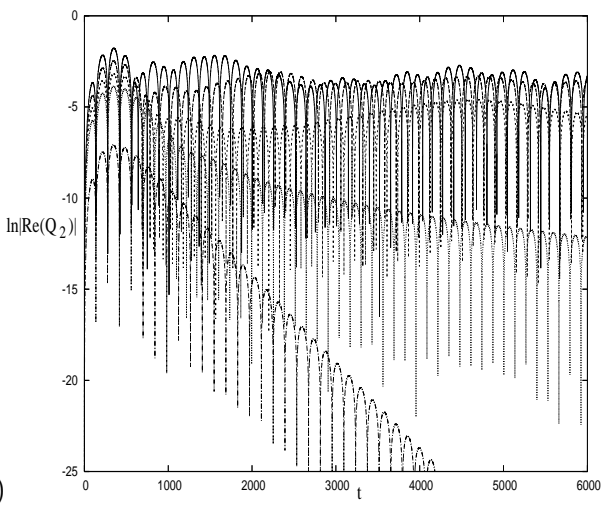

(c)

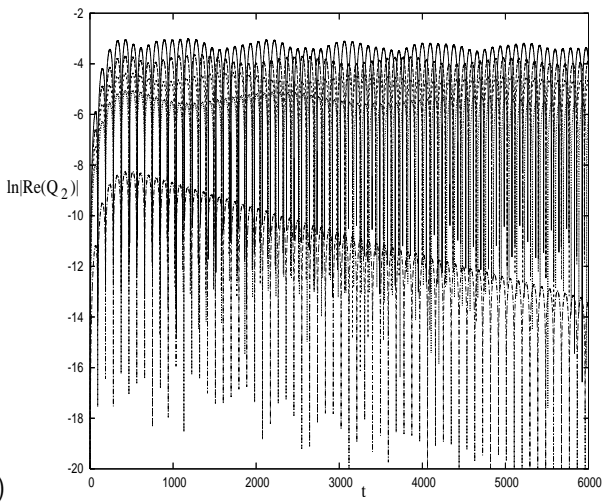

Figure 9. Plot of $\ln \left|\operatorname{Re}\left(Q_{2}\right)\right|$ in the case of the tanh vortex for (a) $\sigma=0.1$, (b) $\sigma=0.5$, (c) $\sigma=0.9$, with $\alpha_{\text {ext }}=\alpha_{\mathrm{qm}}, T=200$ and $R=10^{7}$. In each panel, the curves from bottom to top are for amplitudes $A=0.000413,0.0103,0.0206,0.0413$ and 0.0825 .

then found, giving,

$$
\begin{aligned}
& \sigma=0.1, \quad \gamma_{\mathrm{qm}}=-0.0050, \quad \alpha_{\mathrm{qm}}=0.0108, \quad r_{\mathrm{qm}}=3.02, \\
& \sigma=0.5, \quad \gamma_{\mathrm{qm}}=-0.0048, \quad \alpha_{\mathrm{qm}}=0.0112, \quad r_{\mathrm{qm}}=2.28 \text {, } \\
& \sigma=0.9, \quad \gamma_{\mathrm{qm}}=-0.0010, \quad \alpha_{\mathrm{qm}}=0.0167, \quad r_{\mathrm{qm}}=1.41 .
\end{aligned}
$$

As $\sigma \rightarrow 1$, the radius $r_{\mathrm{qm}}$ tends to the critical radius $\sqrt{2}$ for a top-hat vortex, as expected. From figure 9 , it is clear that threshold for cat's eye formation $A$ decreases in this limit, in agreement with the asymptotic theory of Balmforth et al. (2001). In the limiting case $\sigma=1$ we recover a top-hat vortex, which possesses a normal mode, a Kelvin mode, and the amplitude threshold becomes zero. We also observe the presence of long timescales emerging in these long runs, again in keeping with asymptotic theory, in which the inverse time-scale and amplitude scale with powers of the vorticity gradient $\Omega_{0}^{\prime}\left(r_{\mathrm{qm}}\right)$ at the radius $r_{\mathrm{qm}}$, vanishing in the limit $\sigma \rightarrow 1$.

\section{Landau Poles}

The quasi-modes of a circular vortex have been studied by taking the Laplace transform of the initial value problem for linear, inviscid disturbances to the vortex profile (Briggs et al. 1970; Corngold 1995; Schecter et al. 2000). Using this method, the frequency $-m \alpha_{\mathrm{qm}}$ and the decay rate $\gamma_{\mathrm{qm}}$ appear as the real and imaginary parts of the complex growth rate $-i p_{\mathrm{qm}}$ from a Landau pole at $p_{\mathrm{qm}}$ in the notation below. This gives exponen- 
tial decay as part of the initial transient behaviour of perturbations to the vortex. It is really a cooperative effect of the continuous spectrum, but may be revealed by analytical continuation techniques (Briggs et al. 1970) as a complex frequency at which the Laplace transform of the multipole moment (2.9) is singular. Schecter et al. (2000) discuss how to calculate the Landau pole numerically for a Gaussian vortex in the bounded domain $r \in\left[0, r_{0}\right]$, with the outer boundary condition of constant $\psi$ on $r=r_{0}$.

We re-examine this method in our context, calculate Landau poles in the infinite domain $r \in[0, \infty)$ for different profiles $\Omega_{0}(r)$ and show how these Landau poles relate to the decay rates for $Q_{2}$ that we observed in section $\S 3$. We only sketch the calculations, following closely Briggs et al. (1970) and Schecter et al. (2000), which should be consulted for more details. Linearizing (2.1) about the mean profile $\Omega_{0}(r)$ and $\Psi_{0}(r)$ leads to the following pair of equations

$$
\partial_{t} \hat{\omega}+i m \alpha(r) \hat{\omega}+i m \beta(r) \hat{\psi}=0, \quad \hat{\omega}=-\Delta \hat{\psi}
$$

where $\Delta \equiv \partial_{r}^{2}+r^{-1} \partial_{r}-m^{2} r^{-2}$ and $\hat{\omega}, \hat{\psi}$ are linear perturbations, of mode $m$, to $\Omega_{0}, \Psi_{0}$ respectively. The angular velocity $\alpha(r)$ and vorticity gradient $r \beta(r)$ are defined by

$$
\alpha(r)=-r^{-1} \partial_{r} \Psi_{0}, \quad \beta(r)=r^{-1} \partial_{r} \Omega_{0},
$$

where the mean vorticity and stream function are linked by $\Omega_{0}=-r^{-1} \partial_{r}\left(r \partial_{r} \Psi_{0}\right)$.

We define the Laplace transform pair with respect to $t$ by

$$
\bar{f}(p)=\int_{0}^{\infty} e^{i p t} \hat{f}(t) d t, \quad \hat{f}(t)=-\frac{1}{2 \pi} \int_{\infty+i \delta}^{-\infty+i \delta} e^{-i p t} \bar{f}(p) d p
$$

with any real $\delta>0$. Combining and taking the transform of equations (4.1) leads to an equation for $\bar{\psi}(r, p)$,

$$
\left[\frac{\partial^{2}}{\partial r^{2}}+\frac{1}{r} \frac{\partial}{\partial r}-\frac{m^{2}}{r^{2}}+\frac{m \beta(r)}{p-m \alpha(r)}\right] \bar{\psi}(r, p)=-\frac{i \hat{\omega}(r, 0)}{p-m \alpha(r)} .
$$

The function $Q_{m}$ from (2.9) may be related to the stream function $\hat{\psi}$,

$$
Q_{m}(t)=\lim _{r \rightarrow \infty}\left(m r^{m} \hat{\psi}-r^{m+1} \partial_{r} \hat{\psi}\right) .
$$

In practice one considers an outer boundary $r=r_{0} \gg 1$ and can either impose zero stream function there, $\hat{\psi}=0$, or irrotational flow beyond, $\partial_{r} \hat{\psi}+m r^{-1} \hat{\psi}=0$. For our purposes, both amount to the same for large $r_{0}$ and so we use the former. The $r_{0}$ here is analogous to the position of the wall used by Schecter et al. (2000), but we let $r_{0} \rightarrow \infty$ to produce our results for an infinite domain.

Solving (4.4) by means of the appropriate Green's function (Briggs et al. 1970; Corngold 1995), the Laplace transform of $Q_{m}$ can be written

$$
\bar{Q}_{m}(p)=-\left.\frac{i r^{m+1}}{\Psi_{\mathrm{L}}(r, p)} \int_{0}^{r} \frac{s \Psi_{\mathrm{L}}(s, p) \hat{\omega}(s, 0)}{r(m \alpha(s)-p)} d s\right|_{r=r_{0}}
$$

where the function $\Psi_{\mathrm{L}}(r, p)$ satisfies the homogeneous version of equation (4.4),

$$
\left[\frac{\partial^{2}}{\partial r^{2}}+\frac{1}{r} \frac{\partial}{\partial r}-\frac{m^{2}}{r^{2}}+\frac{m \beta(r)}{p-m \alpha(r)}\right] \Psi_{\mathrm{L}}(r, p)=0,
$$

with $\Psi_{\mathrm{L}}(r, p)=0$ at $r=0$.

If the vorticity profile is smooth and monotonically decreasing on the whole domain $r \in\left[0, r_{0}\right]$, then there are no discrete eigenmodes of the vortex (Briggs et al. 1970). 
(a)

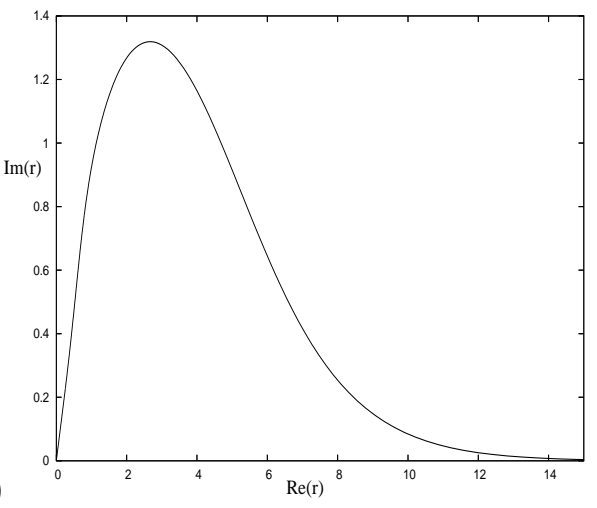

(b)

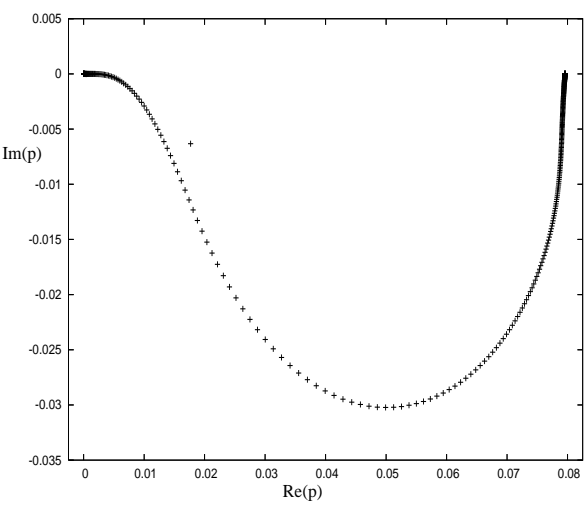

Figure 10. Plot of (a) the contour in the complex $r$ plane given by (4.8) with $\rho_{1}=3 / 2, \rho_{2}=2$ and $\rho_{3}=0$ and (b) the corresponding branch cut in the complex $p$ plane for the Gaussian vortex (2.2). Figure (b) shows a discrete approximation to the continuous spectrum as well as the Landau pole at $p=0.0177-0.0063 i$.

Therefore there are no discrete values of $p$ for which $\Psi_{\mathrm{L}}\left(r_{0}, p\right)=0$, and no poles in $\bar{Q}_{m}(p)$ which would give exponential terms in the inversion of the Laplace transform. However, there is a branch cut along the real $p$ axis in the range $m \alpha\left(r_{0}\right) \leq p \leq m \alpha(0)$.

Using analytic continuation, however, it is possible to deform this branch cut below the real $p$ axis, by deforming the radial contour of integration in (4.6) above the real $r$ axis. If the branch cut is bent sufficiently far below the real $p$ axis, then one or more Landau poles $p=p_{0}$ may appear in the analytic continuation of $\bar{Q}_{m}(p)$ between the branch cut and the real $p$ axis. The is seen in figure 10 for the case for a Gaussian vortex (2.2). When the inversion contour is deformed around such a pole and the branch cut, this leads to a term in $Q_{m}(t)$ of the form $e^{-i p_{0} t}$, which can dominate the early evolution of $Q_{m}(t)$ but which does not correspond to a normal mode; of course $-i p_{0} \equiv-i p_{\mathrm{qm}}$ is the complex quasi-mode growth rate, used first in (3.1).

To track down Landau poles we use contours in the complex $r$ plane of the general form, parameterised by $s$,

$$
r(s)=\tan \left(\frac{\pi}{2}\left[s+i \rho_{1} s \exp \left(\frac{1}{\rho_{2} s^{\rho_{3}}(s-1)}\right)\right]\right),
$$

where $\rho_{1}, \rho_{2}$ and $\rho_{3}$ are constants. Here $s$ is real, in the range $[0,1]$, and the $r$ contour goes from zero to (real) infinity. The form of $\Psi_{\mathrm{L}}(r, p)$ as $r \rightarrow 0$ is $r^{m}$, and so near the origin we impose boundary conditions on (4.7) at $s=\varepsilon \ll 1$,

$$
\Psi_{\mathrm{L}}(r(\varepsilon), p)=r^{m}(\varepsilon), \quad \frac{\partial \Psi_{\mathrm{L}}}{\partial s}(r(\varepsilon), p)=m r^{\prime}(\varepsilon) r^{m-1}(\varepsilon) .
$$

Equation (4.7), with a Gaussian or tanh profile substituted, is then solved by integrating from $s=0$ to $s=1$ along (4.8) by a Runge-Kutta method, and $p$ is updated by Newton's method to make $\Psi_{\mathrm{L}}$ zero at $s=1$. For the contour in (4.8), we use typical values for the constants $\rho_{1}=3 / 2, \rho_{2}=2$ and $\rho_{3}=0$. However the value of $\rho_{3}$ has to be increased when we consider the tanh vortex, because there is an infinite set of branch points of $\alpha_{0}(r)$, given by (2.5), in the complex $r$ plane, which all tend to $r=1$ as $\sigma \rightarrow 1$. In this case the $r$ contour has to be chosen carefully to negotiate these.

Equation (4.7) may also be solved with homogeneous boundary conditions using a global eigenvalue method (Schmid \& Henningson 2001): this was used to obtain figure 10, showing the Landau pole and also an approximation to the continuous spectrum on 
(a)

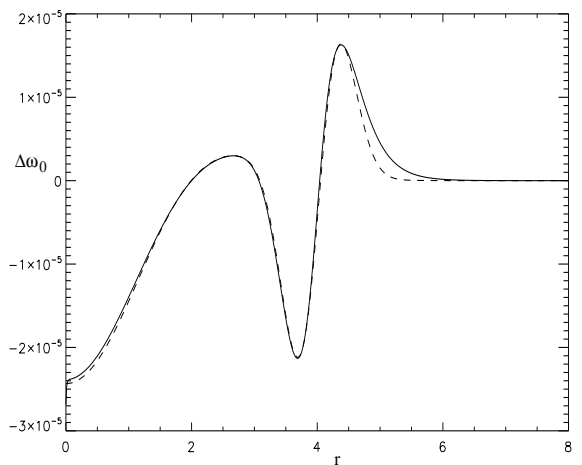

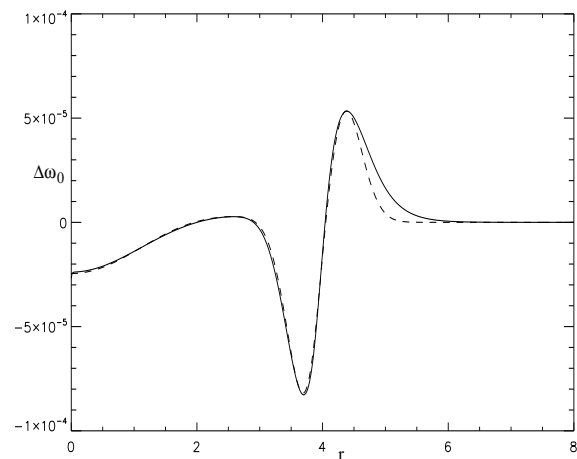

(b)

FIgURE 11. Examples of different modifications $\Delta \omega_{0}$ to the basic vorticity from runs with $R=10^{7}$ and (a) $A=0.00515$ and (b) $A=0.0103$ at $t=3000$. The dashed lines show the approximations discussed in the text.

the distorted branch cut. Both the global and local method have their advantages and disadvantages; however we found that the global method helped to provide a good initial guess for the local method, which then may be used to give a more accurate value for the Landau pole. The results of our computations are given in $(3.1,3.2)$ for the Gaussian vortex and in (3.8) for the tanh family.

From (4.7), the determination of the Landau pole depends on the angular velocity profile $\alpha(r)$ and the gradient of the mean vorticity profile $r \beta(r)=\Omega_{0}^{\prime}(r)$. We found in $\S 3$ that even when a disturbance ultimately decays, the mean vorticity profile is modified during the initial transient, which can move the Landau pole and change the decay rate. Here we show that the modifications found in $\S 3$ give decay rates which agree with the nonlinear code for small $A$. Landau poles are obtained by analytic continuation, and this process is not very stable when applied directly to numerical output from our dynamical runs. Our strategy is to approximate the modified vorticity profile by simple analytical functions, and then to use this in the Landau pole code.

Examples of the modification $\Delta \omega_{0}$ to the profile from $\S 3$ are shown in figure 11 . Note that there is some effect of viscosity visible, giving negative $\Delta \omega_{0}$ at the smaller values of $r$; the Landau pole is not sensitive to this, but it is to the structure near to $r_{\mathrm{qm}}$. We approximate the curves in figure 11 using the family of functions

$$
\Delta \omega_{0}=\left(a_{1} r+b_{1}\right) \exp \left(-\frac{\left(r-c_{1}\right)^{2}}{d_{1}^{2}}\right)+\left(a_{2} r+b_{2}\right) \exp \left(-\frac{\left(r-c_{2}\right)^{2}}{d_{2}^{2}}\right)
$$

where $a_{i}, b_{i}, c_{i}$ and $d_{i}$ are constants. Figure 11(a) shows an example of this approximation as a dashed line. We generally fixed these constants by requiring agreement of the positions of the turning points, and the values of $\Delta \omega_{0}$ at these points. This was done in figure 11(a) but in 11(b) this gave a poor approximation to the gradient at $r_{\mathrm{qm}}$ and a poor estimate of the decay rate. Therefore for figure 11(b), we added to our first fit the function $10^{-5} \times \exp \left(-(r-4.2)^{2} / 0.2^{2}\right)$. This fit, depicted as a dashed line in figure 11(b), gives a better approximation to this gradient, and so an improved value for the Landau pole.

Figure 12 shows a plot of $\ln \left|Q_{2}\right|$ against time $t$ for selected values of the amplitude $A$ for the external angular velocity, $\alpha_{\text {ext }}=\alpha_{\mathrm{qm}}$. Each decay curve (solid) is plotted with the corresponding decay rate obtained from the Landau pole calculation (dash). In each case the vorticity profile is approximated at $t=3000$, except for case 3 where the 


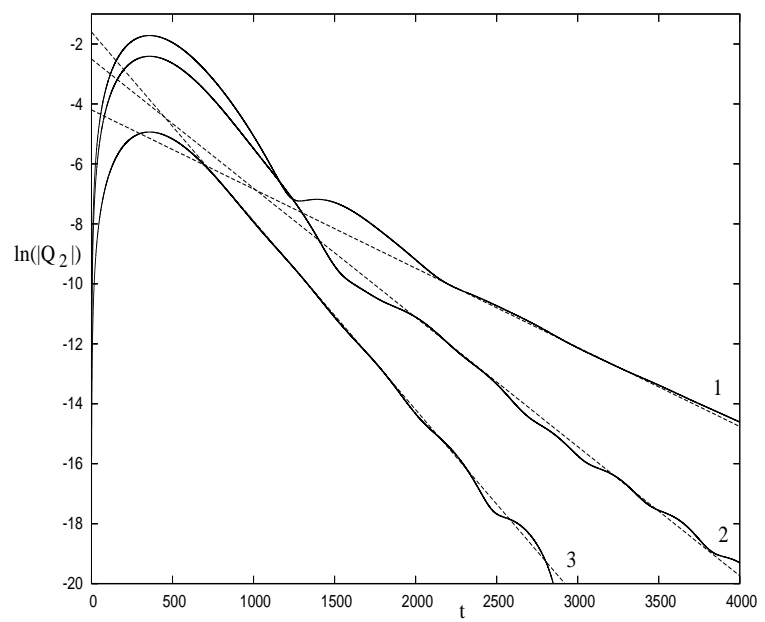

Figure 12. This figure shows $\ln \left|Q_{2}(t)\right|$ (solid), with its corresponding Landau pole decay rate (dash) for $\alpha_{\text {ext }}=\alpha_{\text {ext }}$, and $1: A=0.0103,2: A=0.00515$ and $3: A=0.000413$.

approximation is taken at $t=2000$. The decay rates from the nonlinear code and Landau pole calculations are in satisfactory agreement, with,

$$
\begin{aligned}
& \text { 1: } \quad A=0.0103, \quad \gamma_{Q}(3000)=-0.0024, \quad \gamma_{\mathrm{qm}}=-0.00264 \text {, } \\
& 2: \quad A=0.00515, \quad \gamma_{Q}(3000)=-0.0040, \quad \gamma_{\mathrm{qm}}=-0.00431 \text {, } \\
& 3: \quad A=0.000413, \quad \gamma_{Q}(2000)=-0.0063, \quad \gamma_{\mathrm{qm}}=-0.00630 \text {. }
\end{aligned}
$$

It may be possible to improve the Landau pole method by approximating the vorticity profile more systematically using a series expansion, say of Chebyshev polynomials. This method may give improved results, though the better the approximation on the real line, the more rapid will be the growth of its analytic continuation in the complex plane, which may limit this approach.

\section{Numerical results: varying the external frequency}

In $\S 3.2$ we considered forcing a vortex with an angular velocity equal to that of the quasi-mode. In this section we consider how a Gaussian vortex evolves when the forcing term has an angular velocity $\alpha_{\text {ext }}$ different from $\alpha_{\mathrm{qm}}$. We recall that together with $\alpha_{\text {ext }}$ we can associate a radius $r_{\text {ext }}$, where fluid particles co-rotate and where, at least initially, cat's eyes will tend to form. Of course as the profile changes under dynamical evolution, frequencies and radii can change, particularly at late times when the forcing is switched off. We therefore measure the frequency $\alpha_{Q}$ from $Q_{2}$, and obtain a corresponding resonant radius $r_{Q}$. For an independent estimate of radius we can take the point $r_{\text {cross }}$ at which $\Delta \omega_{0}$ crosses the horizontal axis; see figure 2(b) above, or $13(\mathrm{~b}, \mathrm{~d})$ below. We pose the question: for a given input angular velocity $\alpha_{\text {ext }}$ and radius $r_{\text {ext }}$, where do the cat's eyes form, as measured by $r_{Q}$ and $r_{\text {cross }}$ ?

To begin with, we present a series of runs that parallel those with $\alpha_{\text {ext }}=\alpha_{\mathrm{qm}}$ in figure 2 , but with $\alpha_{\text {ext }}$ larger in figure 13(a,b) and smaller in 13(c,d). In the first pair of panels, the larger value of $\alpha_{\text {ext }}$ corresponds to attempting to generate cat's eyes closer to the origin, at $r_{\text {ext }}=3.3$, and indeed the crossing point $r_{\text {cross }}$ in $13(\mathrm{~b})$ is rather closer to the origin. Note that this crossing point is rather insensitive to the amplitude $A$ : all the curves in 13(b) cross the horizontal axis at approximately the same point. For the second pair 
(a)

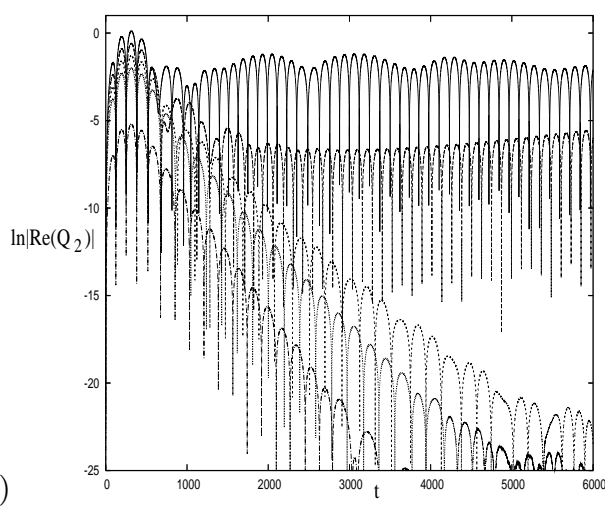

(c)

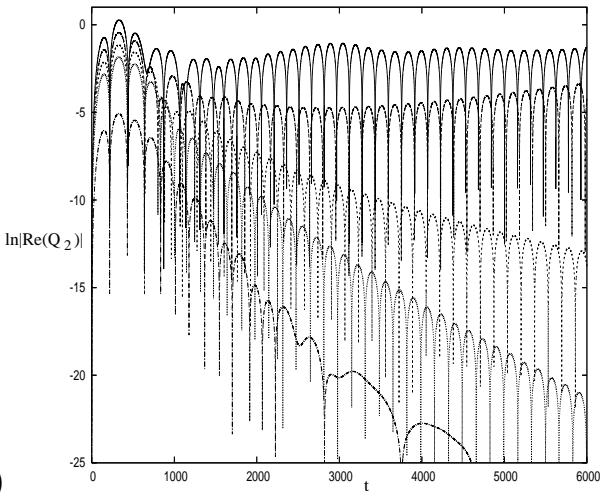

(b)
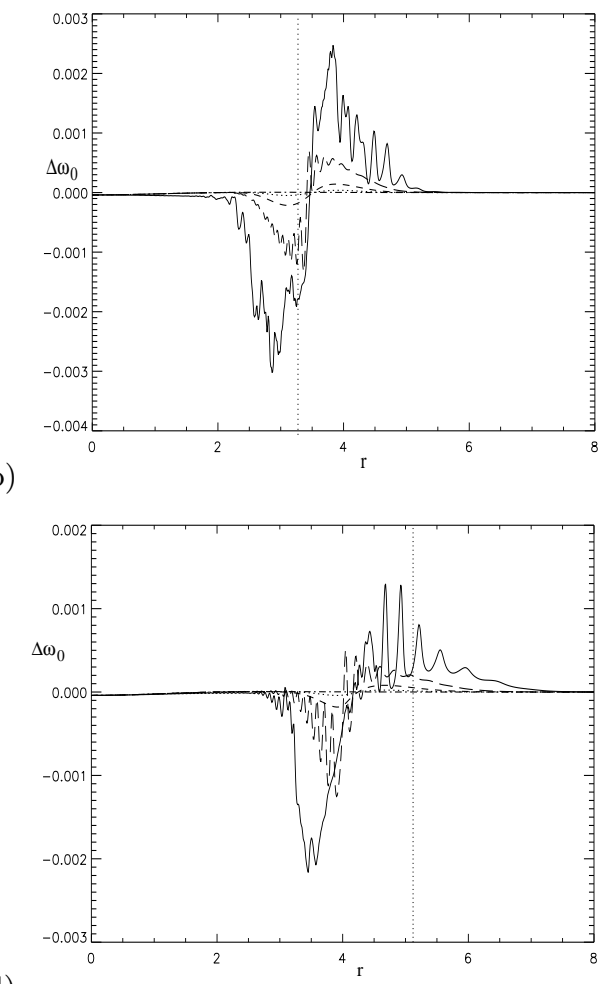

Figure 13. Plot of (a, c) $\ln \left|\operatorname{Re}\left(Q_{2}(t)\right)\right|$ and $(\mathrm{b}, \mathrm{d}) \Delta \omega_{0}$ at $t=5000$, for $T=200, R=10^{7}$ and in $(\mathrm{a}, \mathrm{b}) \alpha_{\text {ext }}=0.014,(\mathrm{c}, \mathrm{d}) \alpha_{\text {ext }}=0.006$. The amplitudes are given by $A=0.000413$ (dot dash), $A=0.0103$ (dot), $A=0.0206$ (dash), $A=0.0413$ (wide dash) and $A=0.0825$ (solid). The vertical dotted line in (b, d) indicates the value of $r_{\text {ext }}$ of (b) 3.3 and (d) 5.2 respectively.

of panels, figure 13(c,d), the small value of $\alpha_{\text {ext }}$ corresponds to larger $r_{\text {ext }}=5.2$; however this appears to be rather larger than the crossing point $r_{\text {cross }}$, which is again insensitive to the amplitude $A$ of the forcing. Thus the behaviour appears different, depending on whether the external frequency $\alpha_{\text {ext }}$ is larger or smaller than the quasi-mode frequency $\alpha_{\mathrm{qm}}$, something we will pick up again shortly.

We note that in general there is a higher amplitude threshold for cat's eye formation in figure 13 than in the $\alpha_{\text {ext }}=\alpha_{\mathrm{qm}}$ case (figure 2), as expected. Again when decay occurs, the decay rate may be modified by structure in the mean profile, and as an example of this we show in figure 14 a comparison of decay rates for the $\alpha_{\text {ext }}=0.014$ high frequency case, and those obtained from a fit of (4.10) to the modification $\Delta \omega_{0}$ of the profile. The numerical values obtained are

$$
\begin{aligned}
& \text { 1: } \quad A=0.0137, \quad \gamma_{Q}(2000)=-0.0044, \quad \gamma_{\mathrm{qm}}=-0.00456, \\
& 2: \quad A=0.0103, \quad \gamma_{Q}(2000)=-0.0049, \quad \gamma_{\mathrm{qm}}=-0.00504 \text {, } \\
& 3: \quad A=0.00515, \quad \gamma_{Q}(2000)=-0.0058, \quad \gamma_{\mathrm{qm}}=-0.00584 \text {. }
\end{aligned}
$$

A plot of $\nu(t)-1$ in figure 15 shows similar results for all three frequencies of $\alpha_{\text {ext }}$ and $A=0.0825$, though with most variability for the high frequency $\alpha_{\text {ext }}$ of curve 2 . In each case, persistent cat's eyes form with $\nu-1$ of the order of a few percent.

To understand where cat's eyes form as a function of the external angular velocity $\alpha_{\text {ext }}$ we now present a series of runs with the external amplitude fixed at a value $A=0.0825$ 


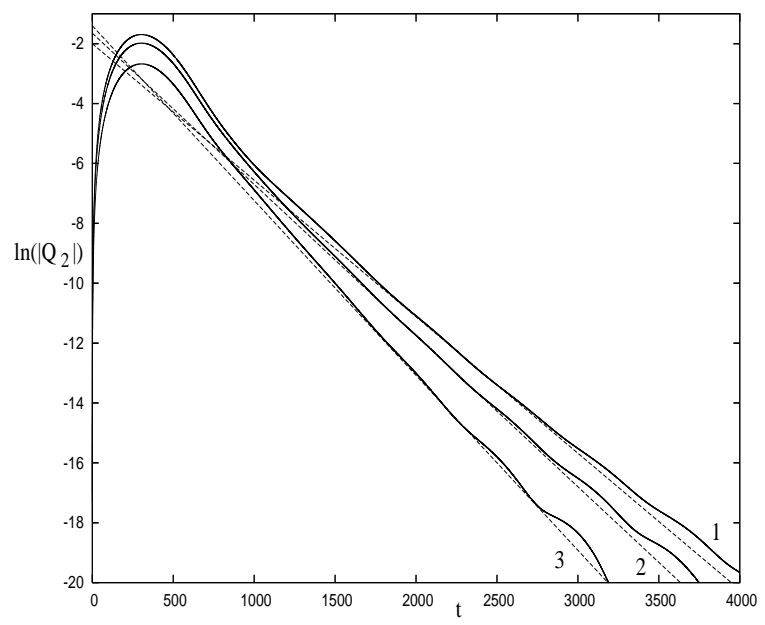

Figure 14. This figure shows $\ln \left|Q_{2}(t)\right|$ (solid), with its corresponding Landau pole decay rate (dash) for $\alpha_{\text {ext }}=0.014$ and $1: A=0.0137,2: A=0.0103$ and $3: A=0.00515$.

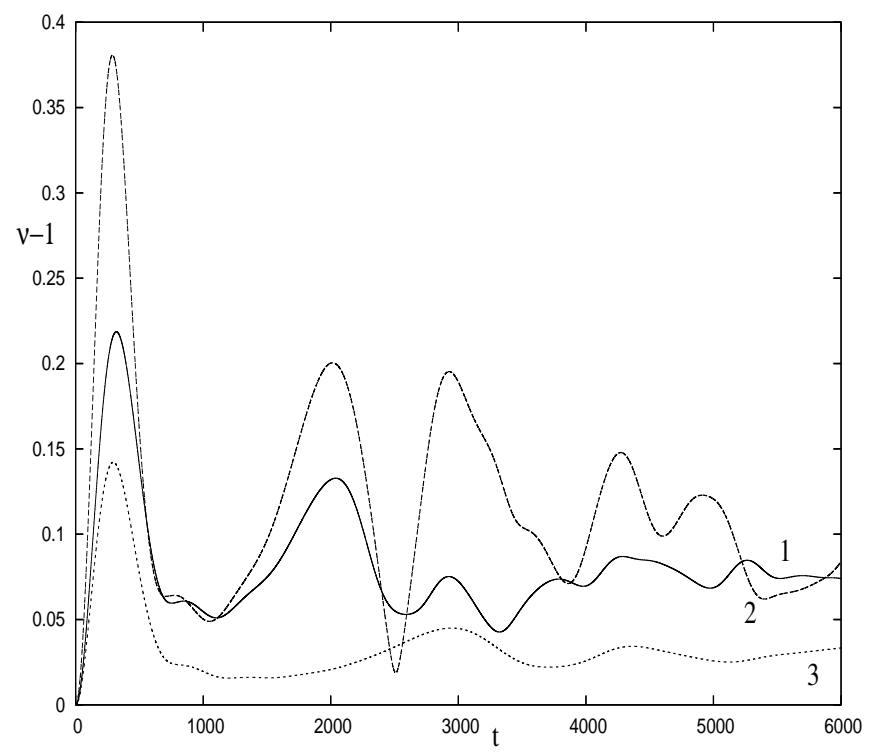

FIGURE 15. Aspect ratio of the streamlines at the origin: $\nu(t)-1$ is shown for $R=10^{7}$, and $1: \alpha_{\mathrm{ext}}=\alpha_{\mathrm{qm}}, 2: \alpha_{\mathrm{ext}}=0.014$ and $3: \alpha_{\mathrm{ext}}=0.006$.

large enough to give cat's eyes for most values of $\alpha_{\text {ext }}$ used; in fact we have persistent cat's eyes for $\alpha_{\text {ext }} \geq 0.003$ for this value of $A$ with $T=200$ and decay for the two data points with $\alpha_{\text {ext }} \leq 0.002$. For each input value of $\alpha_{\text {ext }}$ we measure the final frequency $\alpha_{Q}$ at time $t=5000$ and these results are shown in figure 16(a). On the right of the panel, that is for larger values of $\alpha_{\text {ext }}$ and so $r_{\text {ext }}$ closer to the origin, the angular velocity that emerges is similar to that input, $\alpha_{Q} \simeq \alpha_{\text {ext }}$ (diagonal straight line). On the left-hand side of the panel, at lower external angular velocities and so $r_{\text {ext }}$ further from the origin, the frequency that emerges appears to asymptote at a level similar to that of the quasi-mode, $\alpha_{\mathrm{qm}}$ (horizontal line), as $\alpha_{\text {ext }} \longrightarrow 0$.

Another view is given in the second panel, figure 16(b), which plots various radii 
(a)

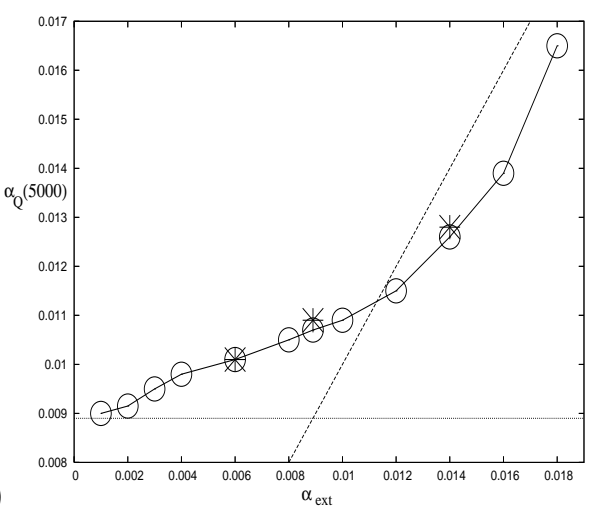

(b)

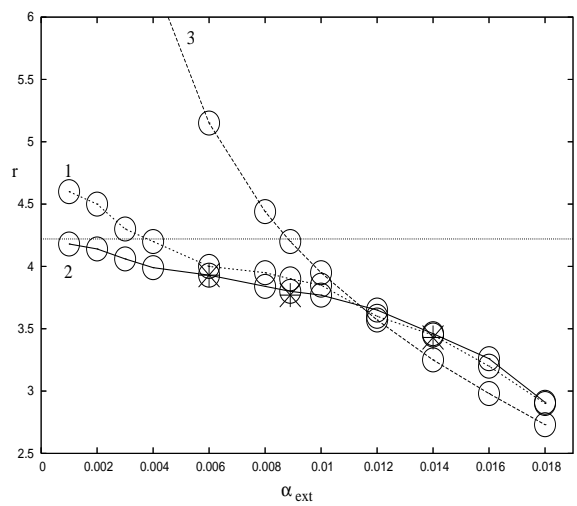

FiguRE 16. Panel (a) shows $\alpha_{Q}$ at $t=5000$ plotted against $\alpha_{\text {ext }}$ with $\alpha_{Q}=\alpha_{\text {qm }}$ marked as a horizontal line and $\alpha_{Q}=\alpha_{\text {ext }}$ as a diagonal line. Panel (b) shows 1: $r_{Q}(5000), 2: r_{\text {cross }}$ and 3: $r_{\text {ext }}$ as a function of $\alpha_{\text {ext }}$. Circles interpolated by straight lines give numerical results, while in each panel the three stars give the results of Landau pole calculations as described in the text.

$r$ against $\alpha_{\text {ext }}$. First we can highlight curve 3 which shows $r_{\text {ext }}$ as a function of $\alpha_{\text {ext }}$ indicating the point that smaller $\alpha_{\text {ext }}$ corresponds to larger radii and vice-versa. The curves 1 and 2 show the two diagnostics, $r_{Q}$ computed from the measured angular velocity $\alpha_{Q}$ using $\alpha_{0}\left(r_{Q}\right)=\alpha_{Q}$, and the crossing point $r_{\text {cross }}$ of the modification to the profile. We note that these two curves generally agree well as a measure of the radius at which cat's eyes form, across the whole range of $\alpha_{\text {ext }}$. (Note that we use $\alpha_{0}(r)$ to translate between radii and frequencies; use of $\alpha(r, t)$ gives similar answers, differing only by a few percent.) For higher angular velocities, to the right of panel 16(b) the three curves are broadly in agreement, and cat's eyes appear to form at the radii linked to $r_{\text {ext }}$, in other words at the radius chosen by the forcing angular velocity $\alpha_{\text {ext }}$. At lower angular velocities however, on the left, curves 1 and 2 tend roughly to the radius $r_{\mathrm{qm}}$ for the quasi-mode; the forcing angular velocity is not relevant and cat's eyes form at $r_{\mathrm{qm}}$ regardless of the external angular velocity imposed.

To understand these differences in behaviour depending on how $\alpha_{\text {ext }}$ compares with $\alpha_{\mathrm{qm}}$ it is worth saying more about the asymptotic model of Balmforth et al. (2001) which gives some insight into what determines the structure, decay rate and frequency of a quasi-mode. These authors use a model in which a compact vortex, that is one with a vorticity distribution vanishing beyond some radius $r_{\mathrm{c}}$, supports an undamped normal mode; one can imagine for example a top-hat vortex supporting a Kelvin mode. This is the normal mode component of the quasi-mode, and has frequency $\alpha_{\mathrm{qm}}$ and critical radius $r_{\mathrm{qm}}>r_{\mathrm{c}}$, associated with the structure of the compact vortex. The authors then add a 'skirt' of weak vorticity, and in the vicinity of $r_{\mathrm{qm}}$, the wind-up of vorticity leads to damping of the normal mode and gives a damping rate $\gamma_{\mathrm{qm}}<0$ proportional to the vorticity gradient in the skirt at $r_{\mathrm{qm}}$. The combination of the normal mode and wind-up of vorticity near $r_{\mathrm{qm}}$ constitute the quasi-mode.

A Gaussian vortex does not have an obvious compact vortex plus skirt structure, but it appears useful to model it qualitatively in these terms (Balmforth et al. 2001). We can informally and very roughly take $r$ less than about 3.5 as the compact vortex core, and larger values of $r$ as falling in the skirt. We can then interpret our results as follows. For low external angular velocities $\alpha_{\text {ext }}$ and so higher values of $r_{\text {ext }}$, the external flow rearranges vorticity in the skirt and excites the quasi-mode structure. But when the external forcing is switched off, the original quasi-mode (whose structure depends on 


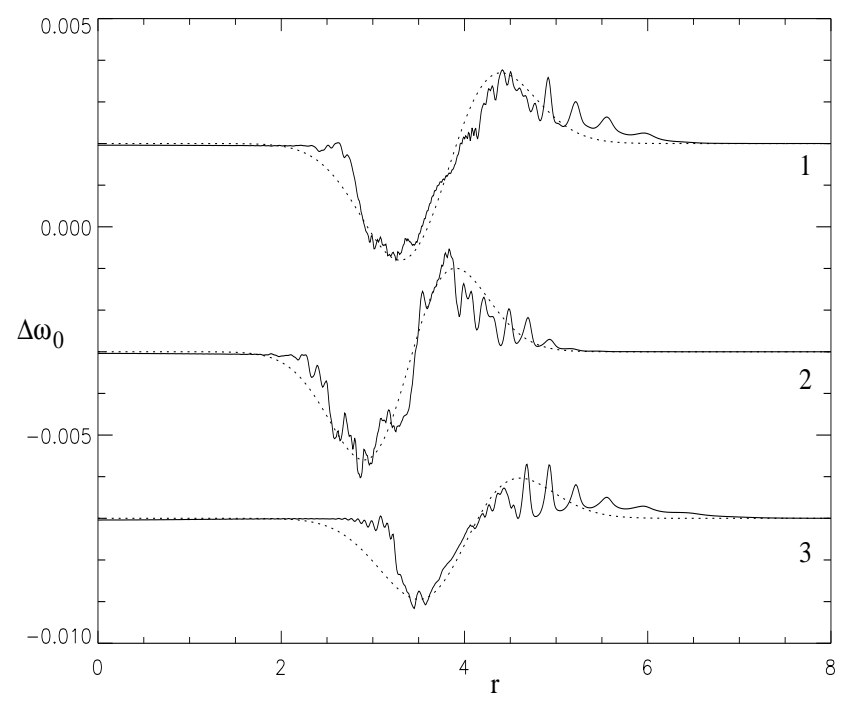

Figure 17. $\Delta \omega_{0}$ (solid) and the approximation using (5.2) (dot) for $A=0.0825$ and $1: \alpha_{\text {ext }}=\alpha_{\mathrm{qm}}, 2: \alpha_{\mathrm{ext}}=0.014$ and $3: \alpha_{\mathrm{ext}}=0.006$. Each result is separated by a constant, and taken at $t=5000$.

the unaffected core of the vortex) takes over and cat's eyes appear at the radius $r_{\mathrm{qm}}$, regardless of the initial $r_{\text {ext }}$.

On the other hand when the external angular velocity is higher, the external flow rearranges vorticity within the compact vortex, closer to the origin. Above the threshold for cat's eye formation it appears to have such a strong effect that the changed profile can now support cat's eyes at the radius $r_{\text {ext }}$ imposed by the forcing. To confirm this interpretation we fitted the modification $\Delta \omega_{0}$ to the profile at $t=5000$ using the form

$$
\Delta \omega_{0}=(a r+b) \exp \left(-\frac{(r-c)^{2}}{d^{2}}\right),
$$

where $a, b, c$ and $d$ are constants. The modifications to the profile give new values for $\alpha_{\mathrm{qm}}$ and $r_{\mathrm{qm}}$, and these may be compared with those measured, $\alpha_{Q}$ and $r_{Q}$ from the Landau pole code. We find

$$
\begin{array}{lllll}
\alpha_{\text {ext }}=0.089, & \alpha_{\mathrm{qm}}=0.0109, & r_{\mathrm{qm}}=3.77, & \alpha_{Q}=0.0107, & r_{Q}=3.80, \\
\alpha_{\text {ext }}=0.014, & \alpha_{\mathrm{qm}}=0.0128, & r_{\mathrm{qm}}=3.43, & \alpha_{Q}=0.0126, & r_{Q}=3.46, \\
\alpha_{\text {ext }}=0.006, & \alpha_{\mathrm{qm}}=0.0101, & r_{\mathrm{qm}}=3.93, & \alpha_{Q}=0.0101, & r_{Q}=3.93 .
\end{array}
$$

The points obtained by the Landau pole code are also shown as stars on figure 16(a) for $\alpha_{Q}$ and on 16(b) for $r_{Q}$. There is good agreement confirming the interpretation given above, despite the relatively crude fitting of the data in figure 17 .

\section{Conclusions}

In this paper we studied how a vortex relaxes after it is subjected to an external forcing which generates cat's eyes inside the vortex. For the Gaussian vortex we mapped out a contour in the $(A, T)$-plane parameterising the forcing, above which cat's eyes are maintained, and below which the vortex relaxes to axisymmetry. This is in agreement 
with the asymptotic model of Balmforth et al. (2001) and the experimental results of Schecter et al. (2000). We computed Landau poles and confirmed that when the vortex does return to axisymmetry, the decay rate of fluctuations is linked to the Landau pole of the linear inviscid problem, and that this decay rate can vary as the mean profile is modified through nonlinear effects during the initial transient. In this sense we have a 'nonlinear' decay rate: nonlinearity in the initial phase controls the decay rate of linear fluctuations in the later phase. We furthermore explored the response of the vortex to varying values of the external angular velocity and its impact on the location of cat's eyes. We find that for angular velocities less than or equal to that of the quasi-mode, we tend to excite just the quasi-mode, and cat's eyes at the radius $r_{\mathrm{qm}}$ with angular velocity $\alpha_{\mathrm{qm}}$. However for external angular velocities greater than $\alpha_{\mathrm{qm}}$ the effect of forcing the vortex is to change the vorticity profile to such an extent that cat's eyes are supported at radii distinct from, and smaller than, that of the quasi-mode $r_{\mathrm{qm}}$. We found that applying the Landau pole calculation to the modified mean profile gave good agreement with the frequency and thus the radius at which cat's eyes form.

We now compare with the work of Le Dizès (2000) who studies the form of steadily rotating cat's eyes embedded in a vortex. His approach is to consider cat's eyes localised in an asymptotically thin critical layer which affects the flow outside through a jump condition (Benney \& Bergeron 1969; Haberman 1972; Brown \& Stewartson 1978; Smith \& Bodonyi 1982). The critical layer may be nonlinear and inviscid, or can be viscous in which case an external forcing is required to maintain a steadily rotating state. The key parameters in this study are the Reynolds number $R$ and a parameter $\varepsilon \ll 1$, which is linked to the aspect ratio of the flow at the origin, with $\nu-1=O(\varepsilon)$, and the cat's eye width, of order $\sqrt{\varepsilon}$. In the case of a Gaussian vortex, with no external strain, the cat's eyes in the study Le Dizès (2000) are localised at a radius $r=3.44$ and have angular velocity $\alpha=0.0127$, in our units, in the inviscid limit $\epsilon^{3} R \gg 1$. This suggests that this angular velocity gives a resonant response to the vortex and that cat's eyes are more likely to persist at this radius.

The key difference between this study and ours is that we have forced the vortex with a transient, relatively high amplitude external flow, which necessarily means the formation of broad cat's eyes, in the cases when they form. The study Le Dizès (2000) involves the limit of infinitesimally thin cat's eyes, and these may only be produced by forcing the vortex at a low amplitude $A$ for a long time $T$, a limit we have not addressed in this paper. The paper Le Dizès (2000) suggests that weak forcing at a frequency $\alpha_{\text {ext }}=0.0127$ should give a resonant response that should lead to the formation of a thin band of cat's eyes for large values of $T$.

To investigate this we have undertaken a few runs at this value of $\alpha_{\text {ext }}$, with some results shown in figure 18, which presents the aspect ratio of the streamlines at the origin for $A=0.0825, T=200$, and $\alpha_{\mathrm{ext}}=0.014,0.0127=\alpha_{\mathrm{LD}}$ and $0.0089=\alpha_{\mathrm{qm}}$. There are minor differences between the results in figure 18; however they are not too significant for this value of $T$. There is no obvious resonant response at $\alpha_{\mathrm{LD}}$, and also we find that a larger amplitude is required to obtain persistent cat's eyes, compared with $\alpha_{\mathrm{qm}}$. In short, for the moderate time-scales of our forcing (and high amplitudes) we do not observe any resonant effects at $\alpha_{\mathrm{LD}}$. However there is preliminary evidence from longer runs (which we do not present here) that for rather larger values of $T$, the threshold for cat's eyes in the case $\alpha_{\mathrm{LD}}$ may drop below that for the case $\alpha_{\mathrm{qm}}$. This issue of how a vortex evolves over long time-scales under weak forcing remains an area of further study. Another topic under investigation is how closely these results may be linked to the tripolar vortices of Rossi et al. (1997). 


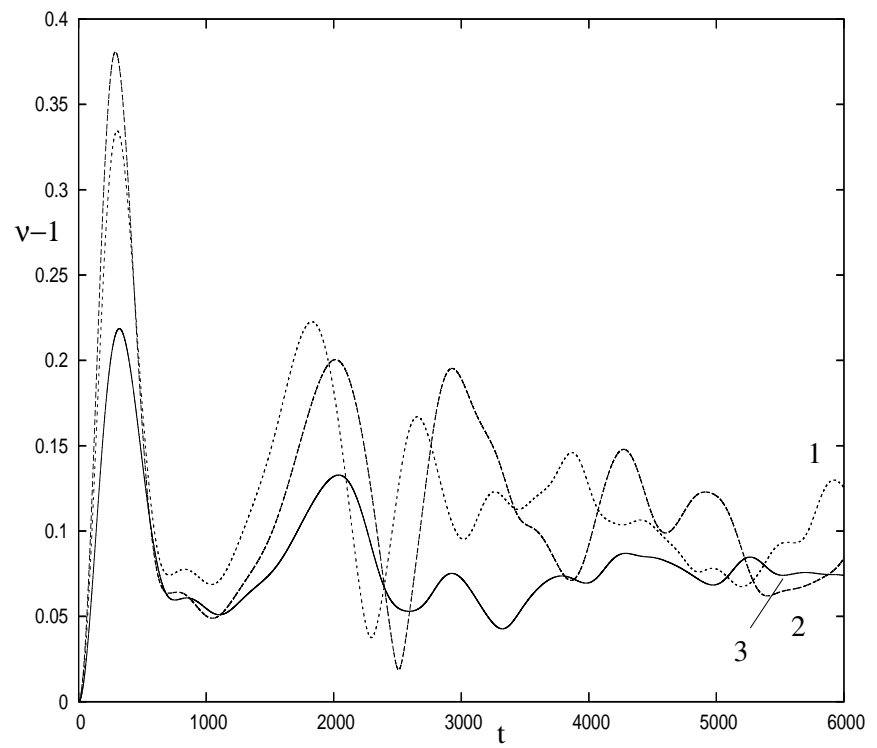

Figure 18. (a) Aspect ratio of the streamlines at the origin: $\nu(t)-1$ is shown for $R=10^{7}$, $A=0.0825, T=200$ and $1: \alpha_{\text {ext }}=\alpha_{\mathrm{LD}}=0.0127,2: \alpha_{\mathrm{ext}}=0.014$ and $3: \alpha_{\mathrm{ext}}=\alpha_{\mathrm{qm}}=0.0089$.

\section{Acknowledgments}

The authors would like to thank Peter Ashwin, Lorena Barba, Andrew Bassom, John Thuburn and anonymous referees for useful and constructive comments. This work is supported by the EPSRC under grant EP/D032202/1.

\section{REFERENCES}

Bajer, K., Bassom, A. P. \& Gilbert, A. D. 2001 Accelerated diffusion in the centre of a vortex. J. Fluid Mech. 437, 395-411.

Balmforth, N. J., Llewellyn Smith, S. G. \& Young, W. R. 2001 Disturbing vortices. J. Fluid Mech. 426, 95-133.

BARBA, L. A. \& LEONARD, A. 2007 Emergence and evolution of tripole vortices from netcirculation initial conditions. Physics of Fluids 19 (1), 017101.

Bassom, A. P. \& Gilbert, A. D. 1998 The spiral wind-up of vorticity in an inviscid planar vortex. J. Fluid Mech. 371, 109-140.

Bassom, A. P. \& Gilbert, A. D. 1999 The spiral wind-up and dissipation of vorticity and a passive scalar in a strained planar vortex. J. Fluid Mech. 398, 245-270.

Benney, D. J. \& Bergeron, R. F. 1969 A new class of nonlinear waves in parallel flows. Stud. Appl. Maths 48, 181-204.

Benzi, R., Paladin, G., Patarnello, S., Santangelo, P. \& Vulpiani, A. 1986 Intermittency and coherent structures in two-dimensional turbulence. J. Phys. A: Math Gen. 19, $3771-3784$.

Bernoff, A. J. \& Lingevitch, J. F. 1994 Rapid relaxation of an axisymmetric vortex. Phys. Fluids 6, 3717-3723.

Brachet, M. E., Meneguzzi, M., Politano, H. \& Sulem, P. L. 1988 The dynamics of freely decaying two-dimensional turbulence. J. Fluid Mech. 194, 333-349.

Briggs, R. J., Daugherty, J. D. \& Levy, R. H. 1970 Role of Landau damping in crossed-field electron beams and inviscid shear flow. Phys. Fluids 13, 421-432. 
Brown, S. N. \& Stewartson, K. 1978 The Evolution of the Critical Layer of a Rossby wave. Part II. Geophys. Astrophys. Fluid Dn. 10, 1-24.

Corngold, N. R. 1995 Linear response of the two-dimensional pure electron plasma: Quasimodes for some model profiles. Phys. Plasmas 2, 620-628.

Dritschel, D. G. 1989 Contour dynamics and contour surgery: numerical algorithms for extended high-resolution modelling of vortex dynamics in two-dimensional, inviscid, incompressible flows. Comput. Phys. Rep. 10, 77-146.

Dritschel, D. G. 1998 On the persistence of non-axisymmetric vortices in inviscid twodimensional flows. J. Fluid Mech. 371, 141-155.

Fornberg, B. 1977 A numerical study of 2-D turbulence. J. Comput. Phys. 25, 1-31.

Guinn, T. A. \& Schubert, W. H. 1993 Hurricane spiral bands. J. Atmos. Sci. 50, 3380-3403.

Haberman, R. 1972 Critical layers in parallel flows. Stud. Appl. Maths 51, 139-161.

Hall, I. M., Bassom, A. P. \& Gilbert, A. D. 2003 The effect of fine structure on the stability of planar vortices. Eur. J. Mech. B Fluids 22 (2), 179-198.

Koumoutsakos, P. 1997 Inviscid Axisymmetrization of an Elliptical Vortex. J. Comput. Phys. $138(2), 821-857$.

LE DizÈs, S. 2000 Non-axisymmetric vortices in two-dimensional flows. J. Fluid Mech. 406, $175-198$.

Legras, B. \& Dritschel, D. 1993 Vortex stripping and the generation of high vorticity gradients in two-dimensional flows. Appl. Sci. Res. 51, 445-455.

Lingevitch, J. F. \& Bernoff, A. J. 1995 Distortion and evolution of a localized vortex in an irrotational flow. Phys. Fluids 7 (5), 1015-1026.

Macaskill, C., Bassom, A. P. \& Gilbert, A. D. 2002 Nonlinear wind-up in a strained planar vortex. Eur. J. Mech. B Fluids 21 (3), 293-306.

MCWilliams, J. C. 1984 The emergence of isolated coherent vortices in turbulent flow. J. Fluid Mech. 146, 21-43.

Melander, M. V., McWilliams, J. C. \& Zabusky, N. J. 1987 Axisymmetrization and vorticity-gradient intensification of an isolated two-dimensional vortex through filamentation. J. Fluid Mech. 178, 137-159.

Moffatt, H. \& KamkaR, H. 1983 On the time-scale associated with flux expulsion. In: Stellar and Planetary Magnetism (Ed. A. M. Soward). Gordon \& Breach Science Publishers pp. 91-97.

Montgomery, M. T. \& Kallenbach, R. J. 1997 A theory for vortex Rossby waves and its application to spiral bands and intensity changes in hurricanes. Q. J. R. Meteorol. Soc. 123, 435-465.

Rhines, P. B. \& Young, W. R. 1983 How rapidly is a passive scalar mixed within closed streamlines? J. Fluid Mech. 133, 133-145.

Rossi, L. F., Lingevitch, J. F. \& Bernoff, A. J. 1997 Quasi-steady monopole and tripole attractors for relaxing vortices. Phys. Fluids 9 (8), 2329-2338.

Schecter, D. A., Dubin, D. H. E., Cass, A. C., Driscoll, C. F., Lansky, I. M. \& O’Neil, T. M. 2000 Inviscid damping of asymmetries on a two-dimensional vortex. Phys. Fluids 12 (10), 2397-2412.

Schmid, P. J. \& Henningson, D. S. 2001 Stability and Transition in Shear Flows. Springer pp. $487-489$.

Smith, F. T. \& Bodonyi, R. J. 1982 Nonlinear critical layers and their development in streaming-flow stability. J. Fluid Mech. 118, 165-185.

Smith, G. B. \& Montgomery, M. T. 1995 Vortex axisymmetrization: Dependence on azimuthal wave-number or asymetric radial structure changes. Q. J. R. Meteorol. Soc. 121, $1615-1650$. 